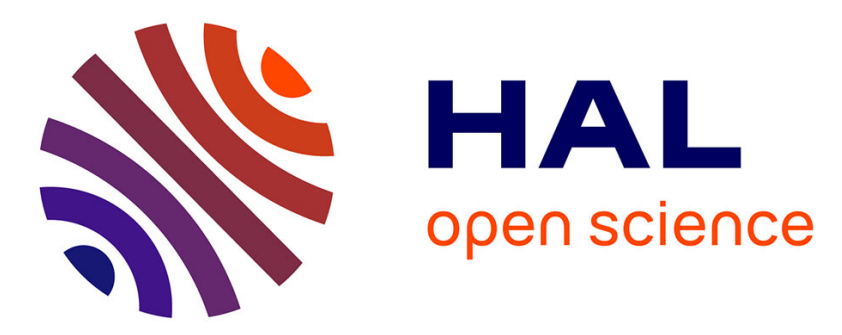

\title{
Stochastic model and generator for random fields with symmetry properties: application to the mesoscopic modeling of elastic random media \\ Johann Guilleminot, Christian Soize
}

\section{- To cite this version:}

Johann Guilleminot, Christian Soize. Stochastic model and generator for random fields with symmetry properties: application to the mesoscopic modeling of elastic random media. Multiscale Modeling and Simulation: A SIAM Interdisciplinary Journal, 2013, 11 (3), pp.840-870. 10.1137/120898346 . hal00854121

\author{
HAL Id: hal-00854121 \\ https://hal.science/hal-00854121
}

Submitted on 26 Aug 2013

HAL is a multi-disciplinary open access archive for the deposit and dissemination of scientific research documents, whether they are published or not. The documents may come from teaching and research institutions in France or abroad, or from public or private research centers.
L'archive ouverte pluridisciplinaire HAL, est destinée au dépôt et à la diffusion de documents scientifiques de niveau recherche, publiés ou non, émanant des établissements d'enseignement et de recherche français ou étrangers, des laboratoires publics ou privés. 


\title{
STOCHASTIC MODEL AND GENERATOR FOR RANDOM FIELDS WITH SYMMETRY PROPERTIES: APPLICATION TO THE MESOSCOPIC MODELING OF ELASTIC RANDOM MEDIA*
}

\author{
J. GUILLEMINOT AND C. SOIZE ${ }^{\dagger}$
}

\begin{abstract}
This paper is concerned with the construction of a new class of generalized nonparametric probabilistic models for matrix-valued non-Gaussian random fields. More specifically, we consider the case where the random field may take its values in some subset of the set of real symmetric positive-definite matrices presenting sparsity and invariance with respect to given orthogonal transformations. Within the context of linear elasticity, this situation is typically faced in the multiscale analysis of heterogeneous microstructures, where the constitutive elasticity matrices may exhibit some material symmetry properties and may then belong to a given subset $\mathbb{M}_{n}^{\text {sym }}(\mathbb{R})$ of the set of symmetric positive-definite real matrices. First of all, we present an overall methodology relying on the framework of information theory and define a particular algebraic form for the random field. The representation involves two independent sources of uncertainties, namely one preserving almost surely the topological structure in $\mathbb{M}_{n}^{\mathrm{sym}}(\mathbb{R})$ and the other one acting as a fully anisotropic stochastic germ. Such a parametrization does offer some flexibility for forward simulations and inverse identification by uncoupling the level of statistical fluctuations of the random field and the level of fluctuations associated with a stochastic measure of anisotropy. A novel numerical strategy for random generation is subsequently proposed and consists in solving a family of Itô stochastic differential equations. The algorithm turns out to be very efficient when the stochastic dimension increases and allows for the preservation of the statistical dependence between the components of the simulated random variables. A Störmer-Verlet algorithm is used for the discretization of the stochastic differential equation. The approach is finally exemplified by considering the class of almost isotropic random tensors.
\end{abstract}

Key words. Random field, Elasticity tensors, Itô stochastic differential equation, Material symmetry, Maximum Entropy principle, Probabilistic model, Random matrix, Verlet scheme.

AMS subject classifications. 74A40, 74B05, 74Q15, 74S60, 65C05, 65C20

1. Introduction. This paper is concerned with the construction of a class of generalized nonparametric probabilistic models for matrix-valued non-Gaussian random fields. More specifically, we consider the case where the random field, denoted by $\{[\boldsymbol{C}(\boldsymbol{x})], \boldsymbol{x} \in \Omega\}$ and indexed by an open bounded domain $\Omega$, may take its values in some subset of the set $\mathbb{M}_{n}^{+}(\mathbb{R})$ of all the symmetric positive-definite $(n \times n)$ real matrices presenting invariance with respect to given orthogonal transformations (without loss of generality, we may assume that $n=3$ or $n=6$ hereinafter). Specifically, such an invariance property is stated as

$$
\forall \boldsymbol{x} \in \Omega,[\boldsymbol{C}(\boldsymbol{x})]=[Q][\boldsymbol{C}(\boldsymbol{x})][Q]^{\mathrm{T}},
$$

in which $[Q]$ is a deterministic $(n \times n)$ real matrix that belongs to a given subspace of the special orthogonal group $\mathrm{SO}\left(n^{\prime}, \mathbb{R}\right.$ ) (which is the group of all the orthogonal $\left(n^{\prime} \times n^{\prime}\right)$ real matrices with unit determinant), with $n^{\prime}=2\left(\right.$ resp. $\left.n^{\prime}=3\right)$ for $n=3$ (resp. $n=6$ ).

In linear elasticity, the random field $\{[\boldsymbol{C}(\boldsymbol{x})], \boldsymbol{x} \in \Omega\}$ may naturally be identified with the random field of apparent properties exhibited by random heterogeneous

${ }^{*}$ This work was supported by the French National Research Agency (ANR) under grants ANR2010-BLAN-0904 and ANR-12-JS09-0001-01.

${ }^{\dagger}$ Université Paris-Est, Laboratoire Modélisation et Simulation Multi Echelle, MSME UMR8208 CNRS, 5 Bd Descartes, 77454 Marne la Vallée, France. Email addresses: johann.guilleminot@univ-paris-est.fr, christian.soize@univ-paris-est.fr. 
microstructures [36]. The consideration of such a random field is of particular interest whenever the underlying microstructure cannot be properly described and reconstructed in a computational framework (this may be the case for biological tissues which often require a hierarchical random morphology to be defined, for instance), or when the usual assumptions of homogenization theories (e.g. scale separation) are not met. The invariance property defined by Eq. (1.1) then gives rise to sparsity and well-known algebraic relationships between some components of the tensor $[\boldsymbol{C}(\boldsymbol{x})]$ [31]. Historically, the characterization of such a random field has been quite extensively addressed by first modeling some morphological parameters of the microstructure (such as the size distribution of the heterogeneities, a $n$-points correlation function describing the spatial distribution of the inclusions and so on; see [41] and [56] for thorough introductions to the field of random microstructure simulations) and then, by proceeding to a hierarchical upscaling towards a coarsest scale (note we do not discuss below the use of multi-scale algorithms and we refer the reader to [60] and the references therein for a survey, among others; see also [4]). When the latter turns out to be (much) smaller than the macroscale - in the usual sense of micromechanics -, one ends up with (realizations of) mesoscale random fields, the definition of which is therefore based upon propagating parameter randomness from one scale to another one. This kind of approach has been successfully applied in the 90's in linear elasticity [34] [35] and was fruitfully revisited since then by using more advanced representation techniques (such as Karhunen-Loève and polynomial chaos expansions) and stochastic solvers (such as the XFEM method [23] and its extension to the stochastic framework - referred to as the XSFEM method [33]- when random geometries are involved), both in the linear [62] [55] and non-linear frameworks [1] [8] [13]. While theoretically appealing, such morphological methods have two important limitations. First of all, they intrinsically depend on the parametric probabilistic description at the microscale, which is intractable in most practical situations unless oversimplifying assumptions are made. Indeed, digitalized experimental realizations of random microstructures are often available in a too small amount and over too small domains to reasonably (i.e. at a sufficiently large level of convergence) identify random fields of morphological characteristics. Besides this consideration, it is worth mentioning that such parametric approaches do not allow the model uncertainties, induced by the choice of the upscaling scheme and its parameters (such as the prescribed boundary conditions; see [24] in elastostatics) for instance, to be accounted for. As far as predictive forward simulations and robust inverse identification are concerned, alternative strategies may then be investigated. In particular, model uncertainties may be also considered by having recourse to the so-called non-parametric framework that has been introduced to take into account both system-parameters and model uncertainties in structural dynamics [45] [46]. Instead of constructing probabilistic models on some microscopic random fields, the approach subsequently involves the definition of probability distributions for mesoscale random fields. It is typically devoted to inverse identification using a limited amount of experimental data (see e.g. [18] [50] for computational examples) and can be used for characterizing the convergence towards the effective properties as the size of the mesoscopic volume increases [49]. The probability distributions are then defined by invoking the information theory introduced by Shannon [42] and more specifically, by combining the theory of random matrices [32] with the maximum entropy (MaxEnt) principle [25] introduced by Jaynes in $1957[5]^{1}$. As the

\footnotetext{
${ }^{1}$ In this work, we consider Shannon's differential entropy for continuous distributions, as opposed to the invariant information measure defined later by Jaynes [26]. This choice is motivated by the
} 
latter requires the definition of some information available on the modeled quantity, the probabilistic models thus constructed are typically referred to as prior probabilistic models. Indeed, the selection of available information defining the constraints integrated in the MaxEnt formulation is a cornerstone of the method, for it aims at ensuring both the mathematical and the physical consistency of the derivations. Undoubtedly, such a process of selection may introduce some discrepancy between the physical random fields and their modeled counterparts. It is also worth noticing that the class thus constructed may be (much) smaller than the class of all the non-Gaussian tensor-valued random fields. Such a drawback may be circumvented in part by combining polynomial chaos representations [61] (see also [12] and [52]) and the prior model (see [50] for the methodology and [51] for the use of Bayesian posteriors on chaos expansion with random coefficients [2]), which seems to be one the most promising way to describe multiscale physical properties from experiments to simulations. Finally, it should be noticed for completeness that chaos representations could have been used for modeling the mesoscale random tensor random field as well (that is, without being coupled with a prior algebraic representation). Such functional models have received a considerable attention during the past two decades, due to the developments of stochastic solvers associated with the now popular stochastic finite element method; see [12] and the recent survey [37]. Although they have been shown to be very useful for uncertainty propagation (through Galerkin-type projections for instance), their identification typically requires a very large amount of experimental data which is seldom (if ever) available in practice.

From a mechanical point of view, mesoscopic elasticity tensors do not exhibit a.s. (almost sure) material symmetries such as those expected or assumed at the macroscale level [35]. Indeed, their stochastic anisotropy measure (defined with respect to a given class of symmetry) presents statistical fluctuations whose level may or may not depend on the level of fluctuations exhibited by the random tensor itself. When the random elasticity tensor is modeled by using the random matrix ensemble $\mathrm{SE}^{+}$defined in [45] [46] (see also §2.3.1), it can be shown that the mean and variance of the anisotropy measure increases almost linearly with the level of fluctuations of the elasticity tensor [21]. This property follows from the fact that each material symmetry can be defined (as a necessary - but not sufficient- condition) by the algebraic multiplicities of the elasticity tensor eigenvalues [40] [6], which are all equal to one for random matrices belonging to $\mathrm{SE}^{+}$and to other classical ensembles (because of the repulsion phenomena [32]). Two types of methods have been proposed so far in the literature for addressing the case when the two levels of fluctuations have to be controlled apart from one another. A first approach consists in prescribing the variances of selected random eigenvalues, in view of the previous definition of the symmetry classes (see [17] and [18] for the random matrix and random field cases, respectively). Such a strategy can be readily applied to all symmetry classes and allows the mean of the anisotropy measure to be imposed within a given range which depends on the mean value and level of statistical fluctuations of the elasticity tensor. An alternative generalized approach for random matrices has been proposed in [53] for the isotropic class and has been generalized to all symmetry classes in [19]. The proposed model is obtained by introducing a particular algebraic representation for

fact that the later involves (and actually depends on) an additional measure - denoted by $m$ in [26], Eq. (63) -, the definition of which is still an open issue. As pointed out in [26], a typical (arbitrary) choice is to take $m$ as a uniform measure, so that the two above entropy definitions may only differ by an irrelevant constant. 
the elasticity tensor, in which an anisotropic germ is combined with a germ taking its values in the set $\mathbb{M}_{n}^{\text {sym }}(\mathbb{R}) \subseteq \mathbb{M}_{n}^{+}(\mathbb{R})$ of elasticity matrices exhibiting the desired material symmetry (hence, the superscript "sym") in a fixed reference coordinates system. By construction, such a generalized approach does not present the limitations induced by the repulsion phenomena but requires further developments in the definition of the probability distributions for $\mathbb{M}_{n}^{\operatorname{sym}}(\mathbb{R})$-valued random variables which exhibit topological zeros (i.e. a.s. sparsity) and a.s. invariance with respect to some orthogonal transformations.

In this work, we build on the results obtained in [19] (for the random matrix case) and address the construction of a class of prior generalized stochastic models for elasticity tensor random fields. The main contributions are the derivation of a stochastic model for non-Gaussian tensor-valued random fields exhibiting some symmetry properties and the construction of a new random generator able to perform in high probabilistic dimensions. The rest of this paper is organized as follows. The overall methodology is first introduced in $\S 2$. We put emphasis on the construction of probability distributions for $\mathbb{M}_{n}^{\text {sym }}(\mathbb{R})$-valued random variables, for which a tensor decomposition is introduced and allows for a systematic derivation for all material symmetry classes. We also discuss strategies for random generation and propose a new algorithm enabling the structure of statistical dependence (induced by the MaxEnt formulation on the family of first-order marginal distributions) to be preserved. In $\S 3$, we illustrate the methodology by considering the class of isotropic tensors.

Notations. For any vectors $\boldsymbol{x}=\left(x_{1}, \ldots, x_{n}\right)$ and $\boldsymbol{y}=\left(y_{1}, \ldots, y_{n}\right)$ in $\mathbb{R}^{n}$, we denote as $\langle\boldsymbol{x}, \boldsymbol{y}\rangle=\sum_{i=1}^{n} x_{i} y_{i}$ the Euclidean inner product in $\mathbb{R}^{n}$ and as $\|\cdot\|$ the associated norm, $\|\boldsymbol{x}\|^{2}=<\boldsymbol{x}, \boldsymbol{x}>$.

The determinant, trace and transpose of matrix $[A] \in \mathbb{M}_{n}(\mathbb{R})\left(\mathbb{M}_{n}(\mathbb{R})\right.$ being the set of all the square $(n \times n)$ real matrices) are denoted by $\operatorname{det}([A]), \operatorname{tr}([A])$ and $[A]^{\mathrm{T}}$, respectively. Let $\mathbb{M}_{n}^{S}(\mathbb{R})$ be the set of all the symmetric $(n \times n)$ real matrices. We denote by $\ll \cdot, \cdot \gg$ the inner product in $\mathbb{M}_{n}^{S}(\mathbb{R})$ such that $\ll[A],[B] \gg=\operatorname{tr}([A][B])$ $\forall([A],[B]) \in \mathbb{M}_{n}^{S}(\mathbb{R}) \times \mathbb{M}_{n}^{S}(\mathbb{R})$. For any random matrix $[\boldsymbol{A}]$ with values in $\mathbb{M}_{n}^{+}(\mathbb{R})$, we further denote by $\delta_{[\boldsymbol{A}]}$ the parameter measuring the level of fluctuations of $[\boldsymbol{A}]$, defined as:

$$
\delta_{[\boldsymbol{A}]}=\sqrt{\mathbb{E}\left\{\|[\boldsymbol{A}]-[\underline{A}]\|_{\mathrm{F}}^{2}\right\} /\|[\underline{A}]\|_{\mathrm{F}}^{2}},
$$

where $\mathbb{E}$ is the mathematical expectation, $\|[A]\|_{\mathrm{F}}^{2}=\operatorname{tr}\left([A]^{2}\right)$ for $[A]$ in $\mathbb{M}_{n}^{+}(\mathbb{R})$ and $[\underline{A}]=\mathbb{E}\{[\boldsymbol{A}]\}$. All inequalities between positive-definite matrices are defined with respect to the usual positive definite (Loewner) ordering.

For any second-rank tensors $[A]$ and $[B], \underline{\bar{\otimes}}$ denotes the usual symmetrized tensor product defined by $2([A] \underline{\bar{\otimes}}[B])_{i j k \ell}=[A]_{i k}[B]_{j \ell}+[A]_{i \ell}[B]_{j k}$.

We adopt the Kelvin matrix representation for fourth-order elasticity tensors [30].

\section{Construction of a class of generalized random field models for elas- ticity tensors.}

2.1. Overview of the methodology. In this paper, we address the construction of a probabilistic model for a second-order elasticity tensor random field, denoted by $\{[\boldsymbol{C}(\boldsymbol{x})], \boldsymbol{x} \in \Omega\}$, with values in $\mathbb{M}_{n}^{+}(\mathbb{R})$, defined on some probability space $(\Theta, \mathcal{T}, P)$ and indexed by an open bounded domain $\Omega$ in $\mathbb{R}^{d}$, with $1 \leq d \leq 3$. We denote by $\boldsymbol{x} \mapsto[\underline{C}(\boldsymbol{x})]$ the mean function of $\{[\boldsymbol{C}(\boldsymbol{x})], \boldsymbol{x} \in \Omega\}$ and assume, for construction purposes, that this mean field is known. In view of inverse identification 
and uncertainty propagation, we may assume that there exists a deterministic matrix $\left[C_{\ell}\right]$ in $\mathbb{M}_{n}^{+}(\mathbb{R})$ bounding almost surely (a.s.) $[\boldsymbol{C}(\boldsymbol{x})]$ from below, for all $\boldsymbol{x}$ in $\Omega$. The consideration of such a lower bound property allows a uniform ellipticity condition (and hence, the well-posedness of the problem; see [3] and [47] for discussions in the scalar and tensor cases) to be recovered and does not affect the construction of the probabilistic model, since it only amounts to construct a stochastic model for the $\mathbb{M}_{n}^{+}(\mathbb{R})$-valued random field $\{[\widetilde{\boldsymbol{C}}(\boldsymbol{x})], \boldsymbol{x} \in \Omega\}$ defined as $[\widetilde{\boldsymbol{C}}(\boldsymbol{x})]=[\boldsymbol{C}(\boldsymbol{x})]-\left[C_{\ell}\right]$ (for all $\boldsymbol{x}$ in $\Omega$ ) and for which the $\mathbb{M}_{n}^{+}(\mathbb{R})$-valued mean function $\boldsymbol{x} \mapsto[\widetilde{\widetilde{C}}(\boldsymbol{x})]$ is given by $[\underline{\widetilde{C}}(\boldsymbol{x})]=[\underline{C}(\boldsymbol{x})]-\left[C_{\ell}\right]$. Whenever required for physical consistency, an upper deterministic bound can be accounted for as well, using the methodology introduced in [16] and [20] (for the random field and random matrix cases respectively; see also [9] for an alternative random matrix formulation).

The inverse problem of constructing a (memoryless or with memory) nonlinear mapping $\mathcal{T}$ such that the stochastic process $\{\mathcal{T}(G(t)), t \geq 0\}$, where $\{G(t), t \geq 0\}$ is a given $\mathbb{R}^{q}$-valued Gaussian stochastic process, has some target probabilistic properties (such as some statistical moments and/or a correlation function) has been discussed quite extensively in the literature (see [11] [14] [15] [39] and the references therein). However, the extension of such approaches to the case of tensor-valued random fields satisfying as many algebraic constraints as the elasticity tensor random field does (e.g. positive-definiteness) seems intractable with the currently available techniques. In this work, we similarly address the construction of a non-Gaussian stochastic model such that the random field $\{[\boldsymbol{C}(\boldsymbol{x})], \boldsymbol{x} \in \Omega\}$ can be expressed through a nonlinear transformation of a Gaussian model and exhibits a family of first-order marginal probability distributions that matches a target family. One may note that:

- As soon as the transformation is defined, the system of all the marginal distributions, which is not explicitly prescribed while constructing the model, is completely defined.

- The random field $\{[\boldsymbol{C}(\boldsymbol{x})], \boldsymbol{x} \in \Omega\}$ inherits a correlation function which is induced by the nonlinear transformation and which depends on the correlation functions retained for the underlying Gaussian model. As mentionned earlier, it is worth pointing out that contrary to what is commonly done or sought in the analysis of stochastic processes, we do not consider information related to a target correlation structure while constructing the transformation.

Thus, the method essentially consists:

1. In considering a particular algebraic decomposition of the non-Gaussian random field $\{[\boldsymbol{C}(\boldsymbol{x})], \boldsymbol{x} \in \Omega\}$, namely:

$$
\forall \boldsymbol{x} \in \Omega, \quad[\boldsymbol{C}(\boldsymbol{x})]=[\mathcal{M}(\boldsymbol{x})]^{1 / 2}[\boldsymbol{A}(\boldsymbol{x})][\mathcal{M}(\boldsymbol{x})]^{1 / 2},
$$

where $\{[\boldsymbol{A}(\boldsymbol{x})], \boldsymbol{x} \in \Omega\}$ and $\{[\boldsymbol{\mathcal { M }}(\boldsymbol{x})], \boldsymbol{x} \in \Omega\}$ are two auxiliary random fields with values in $\mathbb{M}_{n}^{+}(\mathbb{R})$ and $\mathbb{M}_{n}^{\text {sym }}(\mathbb{R})$ respectively (see $\S 2.5$ ). Such an algebraic representation does allow for some flexibility in terms of anisotropy modeling (see [19] [53] for numerical evidences).

2. In introducing two sets of independent second-order centered homogeneous mean-square continuous $\mathbb{R}$-valued Gaussian random fields, denoted by $\left\{\left\{\xi^{\ell}(\boldsymbol{x}), \boldsymbol{x} \in \mathbb{R}^{d}\right\}\right\}_{\ell}$ and $\left\{\left\{\Xi^{\ell}(\boldsymbol{x}), \boldsymbol{x} \in \mathbb{R}^{d}\right\}\right\}_{\ell}$ respectively. Those Gaussian random fields will be referred to as the stochastic germs of the non-Gaussian model.

3. In writing each of the aforementionned auxiliary non-Gaussian (and potentially, non-homogeneous) tensor-valued random fields as a given nonlinear transformation of one of the sets of Gaussian germs. 
This philosophy of construction, pursued (to some extent) in [47] for fully anisotropic tensor-valued non-Gaussian random fields, has motivated the derivations for the random matrix case proposed in [19], which serve as a key ingredient for the extension to the modeling of random fields which is addressed in this work. In $\S 2.2$, we address the definition of the set of the stochastic germs, whereas the definition of the target probability distributions and the construction of the associated random generators will be addressed later in $\S 2.3$ and $\S 2.4$.

2.2. Definition of the set of Gaussian stochastic germs. Throughout this paper, we denote by

$$
\left\{\left\{\xi^{\ell}(\boldsymbol{x}), \boldsymbol{x} \in \mathbb{R}^{d}\right\}\right\}_{\ell=1}^{\ell=n(n+1) / 2}
$$

and

$$
\left\{\left\{\Xi^{\ell^{\prime}}(\boldsymbol{x}), \boldsymbol{x} \in \mathbb{R}^{d}\right\}\right\}_{\ell^{\prime}=1}^{\ell^{\prime}=N}
$$

two sets of independent second-order centered homogeneous real-valued Gaussian random fields, defined on a probability space $(\Theta, \mathcal{T}, P)$, indexed by $\mathbb{R}^{d}$ and with values in $\mathbb{R}$, such that for all $\boldsymbol{x}$ in $\mathbb{R}^{d}$ :

$$
\mathbb{E}\left\{\xi^{\ell}(\boldsymbol{x})^{2}\right\}=1, \quad \mathbb{E}\left\{\Xi^{\ell^{\prime}}(\boldsymbol{x})^{2}\right\}=1 .
$$

These sets of Gaussian random fields are defined by the associated sets $\left\{\mathrm{R}_{\xi^{\ell}}\right\}_{\ell=1}^{\ell=n(n+1) / 2}$ and $\left\{\mathrm{R}_{\Xi^{\ell^{\prime}}}\right\}_{\ell^{\prime}=1}^{\ell^{\prime}=N}$ of normalized autocorrelation functions, which are such that

$$
\forall \ell \in\{1, \ldots, n(n+1) / 2\}, \mathrm{R}_{\xi^{\ell}}(\boldsymbol{y})=\mathbb{E}\left\{\xi^{\ell}(\boldsymbol{x}+\boldsymbol{y}) \xi^{\ell}(\boldsymbol{x})\right\}, \mathrm{R}_{\xi^{\ell}}(0)=1
$$

and

$$
\forall \ell^{\prime} \in\{1, \ldots, N\}, \mathrm{R}_{\Xi^{\ell^{\prime}}}(\boldsymbol{y})=\mathbb{E}\left\{\Xi^{\ell^{\prime}}(\boldsymbol{x}+\boldsymbol{y}) \Xi^{\ell^{\prime}}(\boldsymbol{x})\right\}, \mathrm{R}_{\Xi_{\ell^{\prime}}}(0)=1 .
$$

For later use, we introduce the $\mathbb{R}^{n(n+1) / 2}$-valued and $\mathbb{R}^{N}$-valued Gaussian random fields $\left\{\boldsymbol{\xi}(\boldsymbol{x}), \boldsymbol{x} \in \mathbb{R}^{d}\right\}$ and $\left\{\boldsymbol{\Xi}(\boldsymbol{x}), \boldsymbol{x} \in \mathbb{R}^{d}\right\}$ such that for all $\boldsymbol{x}$ in $\Omega$ :

$$
\boldsymbol{\xi}(\boldsymbol{x})=\left(\xi^{1}(\boldsymbol{x}), \ldots, \xi^{n(n+1) / 2}(\boldsymbol{x})\right), \boldsymbol{\Xi}(\boldsymbol{x})=\left(\Xi^{1}(\boldsymbol{x}), \ldots, \Xi^{N}(\boldsymbol{x})\right) .
$$

It is assumed that the correlation function retained for each stochastic germ is continuous, hence implying that the random fields $\left\{\boldsymbol{\xi}(\boldsymbol{x}), \boldsymbol{x} \in \mathbb{R}^{d}\right\}$ and $\left\{\boldsymbol{\Xi}(\boldsymbol{x}), \boldsymbol{x} \in \mathbb{R}^{d}\right\}$ are mean-square continuous on $\mathbb{R}^{d}$. For a given germ, any type of normalized autocorrelation function can be selected; see the remark below. For computational illustrations, the set of autocorrelation functions that were considered in [47] is used hereinafter. Consequently, each function $\boldsymbol{y} \mapsto \mathrm{R}_{\xi^{\ell}}(\boldsymbol{y})$ is now written as

$$
\mathrm{R}_{\xi^{\ell}}(\boldsymbol{y})=\prod_{j=1}^{d} r_{j}^{\ell}\left(y_{j}\right),
$$

where

$$
\begin{gathered}
r_{j}^{\ell}(0)=1, \\
r_{j}^{\ell}\left(y_{j}\right)=\left(2 \mathcal{L}_{j}^{\ell} /\left(\pi y_{j}\right)\right)^{2} \sin ^{2}\left(\pi y_{j} /\left(2 \mathcal{L}_{j}^{\ell}\right)\right)
\end{gathered}
$$


for $y_{j} \neq 0$ and $\forall j \in\{1, \ldots, d\}, \mathcal{L}_{j}^{\ell}>0$. Note that similar expressions are used for each function $\boldsymbol{y} \mapsto \mathrm{R}_{\Xi^{\ell^{\prime}}}(\boldsymbol{y})$. The power spectral density (PSD) function $\boldsymbol{k} \mapsto \mathrm{S}_{\xi^{\ell}}(\boldsymbol{k})$, defined from $\mathbb{R}^{d}$ into $\mathbb{R}^{+}$and such that

$$
\mathrm{R}_{\xi^{\ell}}(\boldsymbol{y})=\int_{\mathbb{R}^{3}} \exp \{\mathrm{i}<\boldsymbol{y}, \boldsymbol{k}>\} \mathrm{S}_{\xi^{\ell}}(\boldsymbol{k}) \mathrm{d} \boldsymbol{k},
$$

is then given by

$$
\mathrm{S}_{\xi^{\ell}}(\boldsymbol{k})=\prod_{j=1}^{d} s_{j}^{\ell}\left(k_{j}\right),
$$

in which for all $j$ in $\{1, \ldots, d\}$, the function $k_{j} \mapsto s_{j}^{\ell}\left(k_{j}\right)$ is defined as:

$$
s_{j}^{\ell}\left(k_{j}\right)=\left\{\begin{array}{l}
\frac{\mathcal{L}_{j}^{\ell}}{\pi}\left(1-\left|k_{j} \mathcal{L}_{j}^{\ell} / \pi\right|\right) \text { if } \quad\left|k_{j} \mathcal{L}_{j}^{\ell} / \pi\right|<1 . \\
0 \text { otherwise }
\end{array}\right.
$$

It follows that the support Supp $\mathrm{S}_{\xi^{\ell}}$ of $\mathrm{S}_{\xi^{\ell}}$ is compact and is written as

$$
\text { Supp } \mathrm{S}_{\xi^{\ell}}=\left[-\frac{\pi}{\mathcal{L}_{1}^{\ell}}, \frac{\pi}{\mathcal{L}_{1}^{\ell}}\right] \times \ldots \times\left[-\frac{\pi}{\mathcal{L}_{d}^{\ell}}, \frac{\pi}{\mathcal{L}_{d}^{\ell}}\right] \text {. }
$$

Each stochastic germ $\left\{\xi^{\ell}(\boldsymbol{x}), \boldsymbol{x} \in \mathbb{R}^{d}\right\}$ is then entirely defined by the set of $d$ spatial correlation lengths $\left\{\mathcal{L}_{j}^{\ell}\right\}_{j=1}^{j=d}[57]$ such the correlation length associated with the coordinate $x_{j}$ writes:

$$
\int_{0}^{+\infty}\left|\mathrm{R}_{\xi^{\ell}}\left(0, \ldots, y_{j}, \ldots, 0\right)\right| \mathrm{d} y_{j}=\pi s_{j}(0)=\mathcal{L}_{j}^{\ell} .
$$

Eq. (2.11) shows that the support of the PSD function effectively controls the spatial correlation lengths of the random field. Note that the latter do not need to be equal, neither from one direction to another nor from one stochastic germ to another. Further, one should note that the methodology and results provided below hold, regardless of the type of correlation functions which is selected for the germs.

Remark 1. It should be emphasized that the autocorrelation functions are equivalently defined by the associated set of PSD functions, the support of which should preferably be compact for sampling constraints (see [38]). The main properties controlling the spatial correlations turns out to be the normalization (in variance) and the spatial correlation lengths (which are seen to be related to the supports of the PSD functions in the example given above). In addition to these properties, the shape for the normalized autocorrelation functions may be chosen by inferring a target shape from homogenization procedures between the microscale and the mesoscale under consideration (see [35] for instance). Specifically, let us assume that one is concerned with the inverse identification of the set of correlation functions (or equivalently, of the set of power spectral density functions) defining the above Gaussian stochastic germs, so that the correlation function of the constructed random field matches a target function. In the most general setting, one may represent the associated PSD functions by some algebraic functions depending on some parameters (see Chapter XII in [28] for linear filtering techniques of cylindrical Gaussian white noise for Gaussian random fields). We denote by $\boldsymbol{w} \in \mathbb{D}_{\boldsymbol{w}}$ the vector-valued parameter gathering 
all these parameters, with $\mathbb{D}_{\boldsymbol{w}}$ a given admissible set. For any fixed value of $\boldsymbol{w}$, we denote by $[[\mathrm{Cov}]]$ the fourth-order tensor-valued covariance function defined as:

$$
\left[\left[\operatorname{Cov}\left(\boldsymbol{x}, \boldsymbol{x}^{\prime} ; \boldsymbol{w}\right)\right]\right]=\mathbb{E}\left\{\left(\left[\boldsymbol{C}_{\boldsymbol{w}}(\boldsymbol{x})\right]-\left[\underline{C}_{\boldsymbol{w}}(\boldsymbol{x})\right]\right) \otimes\left(\left[\boldsymbol{C}_{\boldsymbol{w}}\left(\boldsymbol{x}^{\prime}\right)\right]-\left[\underline{C}_{\boldsymbol{w}}\left(\boldsymbol{x}^{\prime}\right)\right]\right)\right\}
$$

for any $\left(\boldsymbol{x}, \boldsymbol{x}^{\prime}\right)$ in $\Omega \times \Omega$. In Eq. (2.12), the dependence of $\{[\boldsymbol{C}(\boldsymbol{x})], \boldsymbol{x} \in \Omega\}$ on $\boldsymbol{w}$ is punctually made explicit for consistency - using the subscript " $\boldsymbol{w}$ ". We denote by $\left(\boldsymbol{x}, \boldsymbol{x}^{\prime}\right) \mapsto\left[\left[\widehat{\operatorname{Cov}}\left(\boldsymbol{x}, \boldsymbol{x}^{\prime}\right)\right]\right]$ the target covariance function. An appropriate choice of PSD functions may then be obtained by determining an optimal value $\boldsymbol{w}^{\text {opt }}$ of $\boldsymbol{w}$ such that

$$
\boldsymbol{w}^{\mathrm{opt}}=\underset{\boldsymbol{w} \in \mathbb{D}_{\boldsymbol{w}}}{\arg \min } \mathcal{E}(\boldsymbol{w})
$$

where the $L^{1}$-type cost function $\mathcal{E}$ is defined as

$$
\mathcal{E}(\boldsymbol{w})=\sum_{i \leqslant j, k \leqslant \ell} \int_{\Omega \times \Omega}\left|\left[\left[\widetilde{\operatorname{Cov}}\left(\boldsymbol{x}, \boldsymbol{x}^{\prime}\right)\right]\right]_{i j k \ell}-\left[\left[\operatorname{Cov}\left(\boldsymbol{x}, \boldsymbol{x}^{\prime} ; \boldsymbol{w}\right)\right]\right]_{i j k \ell}\right| \mathrm{d} \boldsymbol{x} \mathrm{d} \boldsymbol{x}^{\prime}
$$

and use has been made of the minor symmetries satisfied by the tensor-valued covariance functions. It is assumed above that [[Cov]] and $\widetilde{\operatorname{Cov}}$ are both integrable on $\Omega \times \Omega$ (if not, the cost-function $\mathcal{E}$ must be adapted accordingly).

When all the correlation functions defining the Gaussian germs have the same algebraic form, numerical results show that the overall shape of the input correlation function is indeed preserved (see $\S 3.2 .2$ and Appendix D). This point is of special interest in practice, since it allows for a substantial reduction of the computational cost associated with the optimization problem defined by Eq. (2.13). Since the Gaussian stochastic germs can be defined through any relevant type of PSD functions, the model certainly offers some flexibility in finding such an optimal parametrization. However, it is worth pointing out that the existence of a solution cannot be ensured in all situations. Clearly, solving this inverse problem in the most general setting is a challenging task that falls out of the scope of this study and is therefore left for future works.

2.3. Construction of a prior probabilistic model for a non-Gaussian random field with anisotropic fluctuations. In this section, we address the construction of a prior stochastic model for a $\mathbb{M}_{n}^{+}(\mathbb{R})$-valued homogeneous non-Gaussian random field $\{[\boldsymbol{A}(\boldsymbol{x})], \boldsymbol{x} \in \Omega\}$. To this aim, we proceed in two steps. First, we construct in $\S 2.3 .1$ a prior probabilistic model for the family $\left\{[A] \mapsto p_{[\boldsymbol{A}(\boldsymbol{x})]}([A] ; \boldsymbol{x})\right\}_{\boldsymbol{x} \in \Omega}$ of first-order marginal p.d.f. of $\{[\boldsymbol{A}(\boldsymbol{x})], \boldsymbol{x} \in \Omega\}$. In $\oint 2.3 .2$, we built on such derivations to explicitly define $\{[\boldsymbol{A}(\boldsymbol{x})], \boldsymbol{x} \in \Omega\}$ as a memoryless nonlinear transformation of the $\mathbb{R}^{n(n+1) / 2}$-valued Gaussian random field $\left\{\boldsymbol{\xi}(\boldsymbol{x}), \boldsymbol{x} \in \mathbb{R}^{d}\right\}$ defined in $\S 2.1$.

2.3.1. Construction of the family of first-order marginal probability distributions. For $\boldsymbol{x}$ fixed in $\Omega$, a prior model for the p.d.f. $[A] \mapsto p_{[\boldsymbol{A}(\boldsymbol{x})]}([A] ; \boldsymbol{x})$ of the $\mathbb{M}_{n}^{+}(\mathbb{R})$-valued random variable $[\boldsymbol{A}(\boldsymbol{x})]$ is defined by invoking the MaxEnt principle under the normalization condition for $p_{[\boldsymbol{A}(\boldsymbol{x})]}$ and the following constraints:

(i) $\mathbb{E}\{[\boldsymbol{A}(\boldsymbol{x})]\}=\left[I_{n}\right]$.

(ii) $\mathbb{E}\{\log (\operatorname{det}([\boldsymbol{A}(\boldsymbol{x})]))\}=\nu_{[\boldsymbol{A}]}(\boldsymbol{x}),\left|\nu_{[\boldsymbol{A}]}(\boldsymbol{x})\right|<+\infty$.

It can be shown that the last constraint implies that both $[\boldsymbol{A}(\boldsymbol{x})]$ and $[\boldsymbol{A}(\boldsymbol{x})]^{-1}$ are second-order random variables (see [45]). Such a construction has been carried out in [45] [46], the set of random matrices thus defined being the so-called normalized 
positive ensemble $\mathrm{SG}^{+}$. After some algebra, the p.d.f. $p_{[\boldsymbol{A}(\boldsymbol{x})]}$ of the random matrix $[\boldsymbol{A}(\boldsymbol{x})]$ can be put in the form:

$$
\begin{aligned}
p_{[\boldsymbol{A}(\boldsymbol{x})]}([A] ; \boldsymbol{x})= & \mathbb{1}_{\mathbb{M}_{n}^{+}(\mathbb{R})}([A]) c_{\boldsymbol{x}}^{\boldsymbol{A}} \operatorname{det}([A])^{(n+1)\left(1-\delta_{[\boldsymbol{A}]}(\boldsymbol{x})^{2}\right) /\left(2 \delta_{[\boldsymbol{A}]}(\boldsymbol{x})^{2}\right)} \\
& \times \exp \left\{-\left((n-1) /\left(2 \delta_{[\boldsymbol{A}]}(\boldsymbol{x})^{2}\right)\right) \operatorname{tr}([A])\right\}
\end{aligned}
$$

in which $c_{\boldsymbol{x}}^{\boldsymbol{A}}$ is the normalization constant (see Eq. (43) of [47] for its closed-form expression) and $\delta_{[\boldsymbol{A}]}(\boldsymbol{x})$ is the dispersion parameter associated with the random variable $[\boldsymbol{A}(\boldsymbol{x})]$ (see Eq. (1.2)). It follows that the family $\left\{p_{[\boldsymbol{A}(\boldsymbol{x})]}(\cdot ; \boldsymbol{x})\right\}_{\boldsymbol{x} \in \Omega}$ of first-order marginal p.d.f. is parametrized by the deterministic field $\boldsymbol{x} \mapsto \delta_{[\boldsymbol{A}]}(\boldsymbol{x})$ controlling the level of statistical fluctuations of the random field $\{[\boldsymbol{A}(\boldsymbol{x})], \boldsymbol{x} \in \Omega\}$.

2.3.2. Random generator and algebraic definition of $\{[\boldsymbol{A}(\boldsymbol{x})], \boldsymbol{x} \in \Omega\}$. In accordance with the properties given in the previous section, the second-order random field $\{[\boldsymbol{A}(\boldsymbol{x})], \boldsymbol{x} \in \Omega\}$ is assumed to belong to the class $\mathrm{SFG}^{+}$of random fields, the construction of which has been carried out in [47]. The ensemble $\mathrm{SFG}^{+}$is an ensemble of homogeneous and normalized non-Gaussian positive-definite matrix-valued random fields, indexed by $\mathbb{R}^{d}$ and with values in the random matrix ensemble $\mathrm{SG}^{+}$introduced in $\S$ 2.3.1. Following [45], the random matrix $[\boldsymbol{A}(\boldsymbol{x})]$ can be decomposed as

$$
\forall \boldsymbol{x} \in \Omega, \quad[\boldsymbol{A}(\boldsymbol{x})]=[\boldsymbol{H}(\boldsymbol{x})]^{\mathrm{T}}[\boldsymbol{H}(\boldsymbol{x})],
$$

wherein $[\boldsymbol{H}(\boldsymbol{x})]$ is an upper-triangular $\mathbb{M}_{n}(\mathbb{R})$-valued random matrix such that for $1 \leq i \leq j \leq n$,

$$
[H(\boldsymbol{x})]_{i j}=\left\{\begin{array}{l}
\frac{\delta_{[\boldsymbol{A}]}(\boldsymbol{x})}{\sqrt{n+1}} \xi^{\alpha_{i j}}(\boldsymbol{x}) \quad \text { if } \quad i<j \\
\frac{\delta_{[\boldsymbol{A}]}(\boldsymbol{x})}{\sqrt{n+1}} \sqrt{2 \mathrm{~F}_{\mathcal{G}\left(\beta_{j}(\boldsymbol{x}), 1\right)}^{-1}\left(\mathrm{~F}_{\mathcal{N}}\left(\xi^{\alpha_{j j}}(\boldsymbol{x})\right)\right)} \quad \text { if } \quad i=j
\end{array}\right.
$$

in which $\alpha_{i j}=i+j(j-1) / 2, \beta_{j}(\boldsymbol{x})=(n+1) /\left(2 \delta_{[\boldsymbol{A}]}(\boldsymbol{x})^{2}\right)+(1-j) / 2, \mathrm{~F}_{\mathcal{G}\left(\beta_{j}(\boldsymbol{x}), 1\right)}^{-1}$ is the Gamma inverse cumulative distribution function with parameters $\beta_{j}(\boldsymbol{x})$ and 1 , and $\mathrm{F}_{\mathcal{N}}$ is the standard normal cumulative distribution function. The probability distributions for the entries of the random matrix $[\boldsymbol{H}(\boldsymbol{x})]$, as defined by Eq. (2.17), result from the use of the MaxEnt principle applied to the random matrix $[\boldsymbol{A}(\boldsymbol{x})]$ (note that the construction is not carried out by considering each component apart from the others), under the constraints stated in $\S 2.5$, and from the change of variable given by Eq. (2.16) (see [45]). Thus, the combination of Eqs. (2.16) and (2.17) defines a memoryless measurable nonlinear mapping $\mathcal{H}$, defined from $\mathbb{R}^{n(n+1) / 2}$ into $\mathbb{M}_{n}^{+}(\mathbb{R})$, such that

$$
[\boldsymbol{A}(\boldsymbol{x})]=\mathcal{H}(\boldsymbol{\xi}(\boldsymbol{x})), \quad \forall \boldsymbol{x} \in \Omega,
$$

where $\left\{\boldsymbol{\xi}(\boldsymbol{x}), \boldsymbol{x} \in \mathbb{R}^{d}\right\}$ is the homogeneous $\mathbb{R}^{n(n+1) / 2}$-valued Gaussian random field defined in $\S 2.1$. Consequently, the random field $\{[\boldsymbol{A}(\boldsymbol{x})], \boldsymbol{x} \in \Omega\}$ inherits a correlation structure which corresponds to the transformation of the correlation structure of $\left\{\boldsymbol{\xi}(\boldsymbol{x}), \boldsymbol{x} \in \mathbb{R}^{d}\right\}$ by the nonlinear transformation $\mathcal{H}$. In practice, independent realizations of the random field $\{[\boldsymbol{A}(\boldsymbol{x})], \boldsymbol{x} \in \Omega\}$ can then be readily obtained from independent realizations of the Gaussian random field $\{\boldsymbol{\xi}(\boldsymbol{x}), \boldsymbol{x} \in \Omega\}$ by combining Eqs. (2.16) and (2.17). It should be noted that the random field $\{[\boldsymbol{A}(\boldsymbol{x})], \boldsymbol{x} \in \Omega\}$ is homogeneous (on $\mathbb{R}^{d}$ for the translation in $\mathbb{R}^{d}$ ) when $\delta_{[\boldsymbol{A}]}(\boldsymbol{x})$ does not depend on $\boldsymbol{x}$ (in which case it is simply denoted as $\delta_{[\boldsymbol{A}]}$ ). 
2.4. Construction of a prior probabilistic model for a random field exhibiting a.s. symmetry properties. Let $\{[\mathcal{M}(\boldsymbol{x})], \boldsymbol{x} \in \Omega\}$ be a non-Gaussian $\mathbb{M}_{n}^{\text {sym }}(\mathbb{R})$-valued random field exhibiting, in some reference frame, some symmetry properties. In order to construct a stochastic model for $\{[\boldsymbol{M}(\boldsymbol{x})], \boldsymbol{x} \in \Omega\}$, we similarly follow a two-step strategy, namely:

- In $\S 2.4 .1$, we are first interested in constructing a prior probabilistic model for the family $\left\{[\mathcal{M}] \mapsto p_{[\mathcal{M}(\boldsymbol{x})]}([\mathcal{M}] ; \boldsymbol{x})\right\}_{\boldsymbol{x} \in \Omega}$ of first-order marginal p.d.f. of $\{[\boldsymbol{M}(\boldsymbol{x})], \boldsymbol{x} \in \Omega\}$. The analysis is performed through subsequent changes of variables, the definitions of which are closely related to random generation constraints and which finally result in the construction of a prior probabilistic model for the family of first-order marginal p.d.f. of an additional random field denoted by $\{\boldsymbol{G}(\boldsymbol{x}), \boldsymbol{x} \in \Omega\}$.

- In $\S 2.4 .3$, we specifically address the construction of a random generator for the random field $\{\boldsymbol{G}(\boldsymbol{x}), \boldsymbol{x} \in \Omega\}$. The construction involves a novel strategy which is based upon solving a family of Itô stochastic differential equations indexed by $\Omega$, for which the family of p.d.f. associated with the family of invariant measures exactly matches the family of MaxEnt p.d.f. constructed in $\S 2.4 .1$. For $\boldsymbol{x}$ fixed in $\Omega$, we then build on the results derived in [48] and we subsequently introduce the spatial dependencies in $\boldsymbol{x}$ through a family $\left\{\boldsymbol{W}_{\boldsymbol{x}}(r), r \geq 0\right\}_{\boldsymbol{x} \in \Omega}$ of $\mathbb{R}^{N}$-valued normalized Wiener processes defined in $\S 2.4 .2$.

2.4.1. Construction of the family of first-order marginal probability distributions. In order to define the stochastic model for the random matrix $[\mathcal{M}(\boldsymbol{x})]$ which exhibits some topological zeros, induced by the material symmetry properties, we first make use of the usual representations for such symmetry properties and decompose the random elasticity matrix as

$$
\forall \boldsymbol{x} \in \Omega, \quad[\mathcal{M}(\boldsymbol{x})]=\sum_{i=1}^{N} M_{i}(\boldsymbol{x})\left[E_{\mathrm{sym}}{ }^{(i)}\right],
$$

in which $\left\{\left[E_{\mathrm{sym}}{ }^{(i)}\right], i=1, \ldots, N\right\}$ (with $2 \leq N \leq 21$ ) is a basis of the matrix space $\mathbb{M}_{n}^{\text {sym }}(\mathbb{R}) \subseteq \mathbb{M}_{n}^{+}(\mathbb{R})$ (see [29] and [59] for the expressions of such basis in a tensor form, among others) and $\left\{\left\{M_{i}(\boldsymbol{x}), \boldsymbol{x} \in \Omega\right\}\right\}_{i=1, \ldots, N}$ is a set of coefficients random fields on this basis. Denoting by $\left\{\boldsymbol{M}(\boldsymbol{x})=\left(M_{1}(\boldsymbol{x}), \ldots, M_{N}(\boldsymbol{x})\right), \boldsymbol{x} \in \Omega\right\}$ the vector-valued random field of the coordinates, it follows that the family of first-order marginal p.d.f. of random field $\{[\boldsymbol{M}(\boldsymbol{x})], \boldsymbol{x} \in \Omega\}$ (indexed by $\Omega$ ) is completely defined by the family $\left\{p_{\boldsymbol{M ( x )}}(\cdot ; \boldsymbol{x})\right\}_{\boldsymbol{x} \in \Omega}$ of first-order marginal p.d.f. of $\{\boldsymbol{M}(\boldsymbol{x}), \boldsymbol{x} \in \Omega\}$. In addition, it should be noted that the a.s. positive-definiteness of $[\mathcal{M}(\boldsymbol{x})]$ implies each element $p_{\boldsymbol{M}(\boldsymbol{x})}$ of the above family to be supported on a subset $\mathcal{C}$, independent of $\boldsymbol{x}$ and possibly unbounded, of $\mathbb{R}^{N}$ :

$$
\mathcal{C}=\left\{\boldsymbol{m} \in \mathbb{R}^{N}, \boldsymbol{m} \neq \mathbf{0} \mid \sum_{i=1}^{N} m_{i}(\boldsymbol{x})\left[E_{\mathrm{sym}}{ }^{(i)}\right]>0\right\} .
$$

In order to handle such a support constraint, we propose to define the random field $\{[\boldsymbol{M}(\boldsymbol{x})], \boldsymbol{x} \in \Omega\}$ through a given nonlinear transformation acting on an auxiliary random field, for which each element of the family of first-order p.d.f. is supported over $\mathbb{R}^{N}$. More specifically, we first introduce the $\mathbb{M}_{n}^{\text {sym }}(\mathbb{R})$-valued random field $\{[\mathcal{N}(\boldsymbol{x})], \boldsymbol{x} \in \Omega\}$ such that

$$
\forall \boldsymbol{x} \in \Omega, \quad[\mathcal{M}(\boldsymbol{x})]=[\underline{\mathcal{M}}(\boldsymbol{x})]^{1 / 2}[\mathcal{N}(\boldsymbol{x})][\underline{\mathcal{M}}(\boldsymbol{x})]^{1 / 2},
$$


where $[\underline{\mathcal{M}}(\boldsymbol{x})]=\mathbb{E}\{[\mathcal{M}(\boldsymbol{x})]\}$. By construction, $\{[\mathcal{N}(\boldsymbol{x})], \boldsymbol{x} \in \Omega\}$ is a normalized random field such that:

$$
\forall \boldsymbol{x} \in \Omega, \quad \mathbb{E}\{[\mathcal{N}(\boldsymbol{x})]\}=\left[I_{n}\right] .
$$

In order to proceed further, we now state the

Proposition 2.1. Let $[N] \in \mathbb{M}_{n}^{\operatorname{sym}}(\mathbb{R})$ and let $\left.\left\{\left[E_{\mathrm{sym}}{ }^{(i)}\right]\right\}_{i=1}^{i=N}\right\}$ be the matrix basis of $\mathbb{M}_{n}^{\text {sym }}(\mathbb{R})$, with $\left[E_{\mathrm{sym}}{ }^{(i)}\right] \in \mathbb{M}_{n}^{S}(\mathbb{R})$ for $i \in\{1, \ldots, N\}$, derived in [59]. Then, there exists a unique symmetric matrix $\left.[G] \in \operatorname{span}\left(\left\{\left[E_{\mathrm{sym}}{ }^{(i)}\right]\right\}_{i=1}^{i=N}\right\}\right)$ such that

$$
[N]=\operatorname{expm}([G]),
$$

where expm denotes the matrix exponential.

Using the above proposition (see Appendix A for the proof), it can be deduced that for any $\boldsymbol{x}$ in $\Omega$, there exists a unique symmetric random matrix $[\mathcal{G}(\boldsymbol{x})]$ such that

$$
\forall \boldsymbol{x} \in \Omega, \quad[\mathcal{N}(\boldsymbol{x})]=\operatorname{expm}([\mathcal{G}(\boldsymbol{x})])
$$

and which exhibits the same topological structure as $[\mathcal{N}(\boldsymbol{x})]$. Equivalently, one has $\forall \boldsymbol{x} \in \Omega,[\mathcal{G}(\boldsymbol{x})]=\operatorname{logm}([\mathcal{N}(\boldsymbol{x})])$, with $\operatorname{logm}$ the matrix logarithm. It should be pointed out that although $[\mathcal{G}(\boldsymbol{x})]$ can be expanded on the same basis,

$$
\forall \boldsymbol{x} \in \Omega, \quad[\mathcal{G}(\boldsymbol{x})]=\sum_{i=1}^{N} G_{i}(\boldsymbol{x})\left[E_{\mathrm{sym}}{ }^{(i)}\right],
$$

it is no longer a positive-definite random matrix. We denote by $\{\boldsymbol{G}(\boldsymbol{x}), \boldsymbol{x} \in \Omega\}$, with $\boldsymbol{G}(\boldsymbol{x})=\left(G_{1}(\boldsymbol{x}), \ldots, G_{N}(\boldsymbol{x})\right)$ for all $\boldsymbol{x}$ in $\Omega$, the $\mathbb{R}^{N}$-valued random field gathering the coordinates random fields of $\{[\mathcal{G}(\boldsymbol{x})], \boldsymbol{x} \in \Omega\}$ onto the matrix basis. In the sequel, we are then interested in the construction of a prior probabilistic model for the family of first-order marginal p.d.f. associated with the random field $\{\boldsymbol{G}(\boldsymbol{x}), \boldsymbol{x} \in \Omega\}$.

As for the anisotropic term, a prior probabilistic model for the random variable $\boldsymbol{G}(\boldsymbol{x})$ ( $\boldsymbol{x}$ being fixed) is sought within the maximum entropy paradigm. We then assume that $[\mathcal{N}(\boldsymbol{x})]$ is a $\mathbb{M}_{n}^{\operatorname{sym}}(\mathbb{R})$-valued random variable satisfying the two following constraints:

$$
\begin{gathered}
\mathbb{E}\{[\mathcal{N}(\boldsymbol{x})]\}=\left[I_{n}\right], \\
\mathbb{E}\{\log (\operatorname{det}([\mathcal{N}(\boldsymbol{x})]))\}=\nu(\boldsymbol{x}),|\nu(\boldsymbol{x})|<+\infty,
\end{gathered}
$$

in which the field $\boldsymbol{x} \mapsto \nu(\boldsymbol{x})$ is assumed to be known at any point $\boldsymbol{x}$ of the domain $\Omega$. Eq. (2.27) implies that both $[\mathcal{N}(\boldsymbol{x})]$ and $[\mathcal{N}(\boldsymbol{x})]^{-1}$ are second-order random variables:

$$
\mathbb{E}\left\{\|[\mathcal{N}(\boldsymbol{x})]\|_{\mathrm{F}}^{2}\right\}<+\infty, \quad \mathbb{E}\left\{\left\|[\mathcal{N}(\boldsymbol{x})]^{-1}\right\|_{\mathrm{F}}^{2}\right\}<+\infty .
$$

It can then be shown that the two above properties imply that $[\mathcal{G}(\boldsymbol{x})]$ is also a secondorder random variable. Upon substituting Eqs. (2.24)-(2.25) in Eqs. (2.26)-(2.27), it can be deduced that the random variable $\boldsymbol{G}(\boldsymbol{x})$ satisfies

$$
\mathbb{E}\left\{\operatorname{expm}\left(\sum_{i=1}^{N} G_{i}(\boldsymbol{x})\left[E_{\mathrm{sym}}{ }^{(i)}\right]\right)\right\}=\left[I_{n}\right]
$$


and

$$
\sum_{i=1}^{N} \mathbb{E}\left\{G_{i}(\boldsymbol{x})\right\} t^{(i)}=\nu(\boldsymbol{x}),|\nu(\boldsymbol{x})|<+\infty,
$$

with $t^{(i)}=\operatorname{tr}\left(\left[E_{\mathrm{sym}}{ }^{(i)}\right]\right)$. It can then be deduced that the MaxEnt-based marginal p.d.f. $\boldsymbol{g} \mapsto p_{\boldsymbol{G}(\boldsymbol{x})}(\boldsymbol{g} ; \boldsymbol{x})$ of $\boldsymbol{G}(\boldsymbol{x})$ at point $\boldsymbol{x}$ writes

$$
\begin{aligned}
p_{\boldsymbol{G}(\boldsymbol{x})}(\boldsymbol{g} ; \boldsymbol{x})= & c_{\boldsymbol{x}}^{\boldsymbol{G}} \exp \left(-\ll\left[\Lambda_{\boldsymbol{x}}^{\text {sol }}\right], \operatorname{expm}\left(\sum_{i=1}^{N} g_{i}\left[E_{\mathrm{sym}}{ }^{(i)}\right] \gg\right.\right. \\
& \left.-\lambda_{\boldsymbol{x}}^{\text {sol }} \sum_{i=1}^{N} g_{i} t^{(i)}\right),
\end{aligned}
$$

where $c_{\boldsymbol{x}}^{\boldsymbol{G}}$ is the normalization constant, $\left[\Lambda_{\boldsymbol{x}}^{\text {sol }}\right]$ and $\lambda_{\boldsymbol{x}}^{\text {sol }}$ are the Lagrange multipliers such that the constraints respectively defined by Eq. (2.29) and Eq. (2.30) are satisfied. A dimensional argument further shows that the Lagrange multiplier $\left[\Lambda_{\boldsymbol{x}}^{\text {sol }}\right]$ must have the same topological form as $[\mathcal{N}(\boldsymbol{x})]$ and can therefore be written as:

$$
\left[\Lambda_{\boldsymbol{x}}^{s o l}\right]=\sum_{i=1}^{N}\left(\lambda_{\boldsymbol{x}}^{s o l}\right)^{(i)}\left[E_{\mathrm{sym}}{ }^{(i)}\right] .
$$

Furthermore, the Lagrange multipliers $\lambda_{\boldsymbol{x}}^{\text {sol }}$ and $\left(\left(\lambda_{\boldsymbol{x}}^{\text {sol }}\right)^{(1)}, \ldots,\left(\lambda_{\boldsymbol{x}}^{\text {sol }}\right)^{(N)}\right)$ belong to some admissible sets, the definition of which ensures the integrability of $p_{\boldsymbol{G}(\boldsymbol{x})}$ (see remark 3 below). For latter use, we let $\lambda_{\boldsymbol{x}}^{\text {sol }}=\left(\left(\lambda_{\boldsymbol{x}}^{\text {sol }}\right)^{(1)}, \ldots,\left(\lambda_{\boldsymbol{x}}^{\text {sol }}\right)^{(N)}, \lambda_{\boldsymbol{x}}^{\text {sol }}\right)$ and we denote by $\mathbb{D}_{\boldsymbol{\lambda}_{\boldsymbol{x}}} \subset \mathbb{R}^{N+1}$ the admissible set of these multipliers. One may also note that the p.d.f. defined by Eq. (2.31) may reduce to a simpler algebraic form whenever the tensor basis exhibits some idempotence and commutativity properties. This is for instance the case of the isotropic class that will be considered in $\S 3$.

Remark 2. Since the associated compliance random field $\left\{[\boldsymbol{C}(\boldsymbol{x})]^{-1}, \boldsymbol{x} \in \Omega\right\}$ shares the same mathematical properties as $\{[\boldsymbol{C}(\boldsymbol{x})], \boldsymbol{x} \in \Omega\}$ - including the tensor decomposition [59] -, it follows that it can also be modeled and generated using the proposed stochastic representation and algorithms.

Remark 3. The proposed methodology essentially consists in relaxing the support constraint (to be used in the MaxEnt approach) by introducing the change of variable $[\mathcal{N}(\boldsymbol{x})] \mapsto[\mathcal{G}(\boldsymbol{x})]=\operatorname{logm}([\mathcal{N}(\boldsymbol{x})])$ ( $\boldsymbol{x}$ being fixed in $\Omega$ ), while preserving the available information on $[\mathcal{N}(\boldsymbol{x})]$. The equivalence between the two constructions is therefore worth investigating, and one may wonder whether or not it is equivalent to construct a probabilistic model for the random matrix $[\mathcal{N}(\boldsymbol{x})]$ under the constraints given by Eqs. (2.26)-(2.27), together with the support information (i.e. $[\mathcal{N}(\boldsymbol{x})] \in \mathbb{M}_{n}^{+}(\mathbb{R})$ a.s.), or to define $[\mathcal{N}(\boldsymbol{x})]$ as $[\mathcal{N}(\boldsymbol{x})]=\operatorname{expm}([\mathcal{G}(\boldsymbol{x})])$, the prior model for $[\mathcal{G}(\boldsymbol{x})]$ being constructed under the constraints given by Eqs.(2.29)-(2.30). In order to comment on this issue, let us derive the p.d.f. of $[\mathcal{N}(\boldsymbol{x})]$ induced by the proposed construction. The jacobian $J$ of the matrix transformation $\mathbb{M}_{n}^{+}(\mathbb{R}) \ni[N] \mapsto[G]=\operatorname{logm}([N]) \in \mathbb{M}_{n}^{S}(\mathbb{R})$ reads as

$$
J=\left(\prod_{i=1}^{n} \frac{1}{\rho_{i}}\right)\left(\prod_{i<j}\left|\frac{\log \left(\rho_{j}\right)-\log \left(\rho_{i}\right)}{\rho_{j}-\rho_{i}}\right|\right)
$$


where $\rho_{1}<\ldots<\rho_{n}$ are the strictly positive real eigenvalues of $[N]$. It can then be deduced from Eq. (2.31) that the p.d.f. of $[\mathcal{N}(\boldsymbol{x})]$ writes, after some algebra:

$$
\begin{aligned}
p_{[\mathcal{N}(\boldsymbol{x})]}([N] ; \boldsymbol{x})= & \underbrace{\mathbb{1}_{\mathbb{M}_{n}^{+}(\mathbb{R})}([N]) c_{\boldsymbol{x}}^{N} \operatorname{det}([N])^{-\lambda_{\boldsymbol{x}}^{s o l}-1} \exp \left(-\ll\left[\Lambda_{\boldsymbol{x}}^{\text {sol }}\right],[N] \gg\right)}_{(\star)} \\
& \times \underbrace{\prod_{i<j}\left|\frac{\log \left(\rho_{i}\right)-\log \left(\rho_{j}\right)}{\rho_{i}-\rho_{j}}\right|}_{(\star \star)},
\end{aligned}
$$

Formally, the first part of the above p.d.f., denoted by $(\star)$, coincides with the form that would be obtained by constructing the prior stochastic model for $[\mathcal{N}(\boldsymbol{x})]$, taking into account both the support constraint and Eqs. (2.26)-(2.27). The second part $(\star \star)$ arises from the change of variable and shows that the two constructions are not exactly equivalent to each other. Upon introducing a further change of variable between $[\mathcal{N}(\boldsymbol{x})]$ and its random eigenvalues and eigenvectors, it can finally be shown that there exists an admissible range for parameter $\lambda_{\boldsymbol{x}}^{\text {sol }}$ such that the p.d.f. defined by Eq. (2.34) is integrable (see Appendix B).

2.4.2. Definition of a family of normalized Wiener processes. Let $\boldsymbol{W}=$ $\left\{\boldsymbol{W}_{\boldsymbol{x}}(r)=\left(W_{\boldsymbol{x}}^{(1)}(r), \ldots, W_{\boldsymbol{x}}^{(N)}(r)\right), \boldsymbol{x} \in \Omega, r \in \mathbb{R}^{+}\right\}$be a $\mathbb{R}^{N}$-valued centered secondorder Gaussian random field such that:

- for all $\boldsymbol{x}$ in $\Omega, \boldsymbol{W}_{\boldsymbol{x}}(0)=\mathbf{0}$ a.s.;

- the generalized time-derivative (i.e. in the sense of generalized stochastic processes) $\mathrm{D}_{t} \boldsymbol{W}$ of $\boldsymbol{W}$ is the cylindrical normalized Gaussian white noise $\boldsymbol{B}$ (see e.g. Chapters XI and XII in [28] - see in particular the sections XII.4.3 and XII.4.5).

The covariance generalized function $\left[\mathrm{C}_{\boldsymbol{B}}\right]$ of $\boldsymbol{B}$ then reads

$$
\forall\left(\boldsymbol{x}, \boldsymbol{x}^{\prime}\right) \in \Omega \times \Omega, \quad \forall \tau \in \mathbb{R}, \quad\left[\mathrm{C}_{\boldsymbol{B}}\left(\boldsymbol{x}, \boldsymbol{x}^{\prime}, t+\tau, t\right)\right]=\delta_{0}(\tau)\left[\mathrm{R}_{\boldsymbol{B}}\left(\boldsymbol{x}, \boldsymbol{x}^{\prime}\right)\right],
$$

where $\delta_{0}$ is the Dirac generalized function at the origin of $\mathbb{R}$. In Eq. (2.35), $\left[\mathrm{R}_{\boldsymbol{B}}\right]$ denotes the continuous $\mathbb{M}_{N}(\mathbb{R})$-valued function defined on $\Omega \times \Omega$ as

$$
\forall 1 \leqslant \ell, \ell^{\prime} \leqslant N, \quad\left[\mathrm{R}_{\boldsymbol{B}}\left(\boldsymbol{x}, \boldsymbol{x}^{\prime}\right)\right]_{\ell \ell^{\prime}}:=\delta_{\ell \ell^{\prime}} \mathrm{R}_{\Xi^{\ell^{\prime}}}\left(\boldsymbol{x}-\boldsymbol{x}^{\prime}\right),
$$

where $\delta_{\ell \ell^{\prime}}$ is the Kronecker delta and $\mathrm{R}_{\Xi^{\ell^{\prime}}}$ is the correlation function of Gaussian random field $\left\{\Xi^{\ell^{\prime}}(\boldsymbol{x}), \boldsymbol{x} \in \mathbb{R}^{d}\right\}$ defined in $\S 2.2$. Consequently, for all $\boldsymbol{x}$ fixed in $\Omega$, $\boldsymbol{W}_{\boldsymbol{x}}=\left\{\boldsymbol{W}_{\boldsymbol{x}}(r), r \geq 0\right\}$ is a normalized $\mathbb{R}^{N}$-valued Wiener process, that is, $\left\{\boldsymbol{W}_{\boldsymbol{x}}(r), r \geq\right.$ $0\}$ satisfies the following properties:

- the real-valued stochastic processes $W_{\boldsymbol{x}}^{(1)}, \ldots, W_{\boldsymbol{x}}^{(N)}$ are mutually independent;

- one has $\boldsymbol{W}_{\boldsymbol{x}}(0)=\mathbf{0}$ a.s.;

- the process $\boldsymbol{W}_{\boldsymbol{x}}$ has independent increments;

- for all $0 \leqslant s<t<+\infty$, the increment $\Delta \boldsymbol{W}_{\boldsymbol{x}}^{s t}:=\boldsymbol{W}_{\boldsymbol{x}}(t)-\boldsymbol{W}_{\boldsymbol{x}}(s)$ is a $\mathbb{R}^{N_{-}}$ valued second-order random variable which is Gaussian, centered and with covariance matrix $\left[\mathrm{C}_{\Delta \boldsymbol{W}_{\boldsymbol{x}}^{s t}}\right]=(t-s)\left[I_{N}\right]\left(\right.$ with $\left[I_{N}\right]$ the $(N \times N)$ identity matrix).

In the next two sections, we make use of such a construction to derive a new random generation algorithm for random field $\{\boldsymbol{G}(\boldsymbol{x}), \boldsymbol{x} \in \Omega\}$. 
2.4.3. Random generator and definition of $\{\boldsymbol{G}(\boldsymbol{x}), \boldsymbol{x} \in \Omega\}$. For $\boldsymbol{\lambda}_{\boldsymbol{x}}$ fixed in $\mathbb{D}_{\boldsymbol{\lambda}_{\boldsymbol{x}}}$, we denote by $\Phi$ the potential function defined from $\mathbb{R}^{N}$ into $\mathbb{R}$ by:

$$
\Phi\left(\boldsymbol{u} ; \boldsymbol{\lambda}_{\boldsymbol{x}}\right)=\ll \sum_{i=1}^{N} \lambda_{\boldsymbol{x}}^{(i)}\left[E_{\mathrm{sym}}{ }^{(i)}\right], \operatorname{expm}\left(\sum_{i=1}^{N} u_{i}\left[E_{\mathrm{sym}}{ }^{(i)}\right]\right) \gg+\lambda_{\boldsymbol{x}} \sum_{i=1}^{N} u_{i} t^{(i)} .
$$

Let $\boldsymbol{Z}_{\boldsymbol{\lambda}_{\boldsymbol{x}}}$ be the $\mathbb{R}^{N}$-valued random variable defined by the p.d.f. $p_{\boldsymbol{\lambda}_{\boldsymbol{x}}}: \mathbb{R}^{N} \rightarrow \mathbb{R}^{+}$ given by

$$
\forall \boldsymbol{u} \in \mathbb{R}^{N}, \quad p_{\boldsymbol{\lambda}_{\boldsymbol{x}}}(\boldsymbol{u} ; \boldsymbol{x})=c_{\boldsymbol{\lambda}_{\boldsymbol{x}}} \exp \left(-\Phi\left(\boldsymbol{u} ; \boldsymbol{\lambda}_{\boldsymbol{x}}\right)\right),
$$

where $c_{\boldsymbol{\lambda}_{\boldsymbol{x}}}$ is the normalization constant. Consequently, one has

$$
\forall \boldsymbol{g} \in \mathbb{R}^{N}, \quad p_{\boldsymbol{G}(\boldsymbol{x})}(\boldsymbol{g} ; \boldsymbol{x})=p_{\boldsymbol{\lambda}_{\boldsymbol{x}}^{\text {sol }}}(\boldsymbol{g} ; \boldsymbol{x}),
$$

with $c_{\boldsymbol{\lambda}_{\boldsymbol{x}}^{s o l}}=c_{\boldsymbol{x}}^{G}$, and

$$
\boldsymbol{G}(\boldsymbol{x})=Z_{\lambda_{\boldsymbol{x}}^{\text {sol }}}
$$

for the convergence in probability distribution.

For $\boldsymbol{x}$ fixed in $\Omega$, let $\left\{\left(\boldsymbol{U}_{\boldsymbol{x}}(r), \boldsymbol{V}_{\boldsymbol{x}}(r)\right), r \in \mathbb{R}^{+}\right\}$be a Markov stochastic process, defined on probability space $(\Theta, \mathcal{T}, \mathcal{P})$, with values in $\mathbb{R}^{N} \times \mathbb{R}^{N}$ and satisfying the following Itô stochastic differential equation (ISDE):

$$
\forall r \in \mathbb{R}^{+},\left\{\begin{array}{l}
\mathrm{d} \boldsymbol{U}_{\boldsymbol{x}}(r)=\boldsymbol{V}_{\boldsymbol{x}}(r) \mathrm{d} r \\
\mathrm{~d} \boldsymbol{V}_{\boldsymbol{x}}(r)=-\nabla_{\boldsymbol{u}} \Phi\left(\boldsymbol{U}_{\boldsymbol{x}}(r) ; \boldsymbol{\lambda}_{\boldsymbol{x}}\right) \mathrm{d} r-\frac{f_{\boldsymbol{x}}^{0}}{2} \boldsymbol{V}_{\boldsymbol{x}}(r) \mathrm{d} r+\sqrt{f_{\boldsymbol{x}}^{0}} \mathrm{~d} \boldsymbol{W}_{\boldsymbol{x}}(r)
\end{array},\right.
$$

wherein $\left\{f_{\boldsymbol{x}}^{0}\right\}_{\boldsymbol{x} \in \Omega}$ is a family of free $\mathbb{R}^{+}$-valued parameters and $\left\{\boldsymbol{W}_{\boldsymbol{x}}(r), r \geq 0\right\}$ is the $\mathbb{R}^{N}$-valued normalized Wiener process defined in $\S 2.4 .2$. The initial conditions associated with Eq. (2.41) are given by $\boldsymbol{U}_{\boldsymbol{x}}(0)=\boldsymbol{U}_{\boldsymbol{x}}^{0}$ and $\boldsymbol{V}_{\boldsymbol{x}}(0)=\boldsymbol{V}_{\boldsymbol{x}}^{0}$ a.s., where the probability distribution $P_{\boldsymbol{U}_{\boldsymbol{x}}^{0}, \boldsymbol{V}_{\boldsymbol{x}}^{0}}(\mathrm{~d} \boldsymbol{u}, \mathrm{d} \boldsymbol{v} ; \boldsymbol{x})$ of $\left(\boldsymbol{U}_{\boldsymbol{x}}^{0}, \boldsymbol{V}_{\boldsymbol{x}}^{0}\right)$ is independent of the stochastic process $\left\{\boldsymbol{W}_{\boldsymbol{x}}(r), r \geq 0\right\}$ and does not need to be equal to the invariant measure. If deterministic initialization vectors $\left(\boldsymbol{u}_{\boldsymbol{x}}^{0}, \boldsymbol{v}_{\boldsymbol{x}}^{0}\right)$ are used, then $P_{\boldsymbol{U}_{\boldsymbol{x}}^{0}, \boldsymbol{V}_{\boldsymbol{x}}^{\mathrm{o}}}(\mathrm{d} \boldsymbol{u}, \mathrm{d} \boldsymbol{v} ; \boldsymbol{x})=\delta_{0}\left(\boldsymbol{u}-\boldsymbol{u}_{\boldsymbol{x}}^{0}\right) \otimes \delta_{0}\left(\boldsymbol{v}-\boldsymbol{v}_{\boldsymbol{x}}^{0}\right)$. Let us first assume the above ISDE admits a unique solution that is defined almost surely for all $r \geq 0$, implying that there is no explosion of the solution (see the Theorems 4 and 5 in Chapter 9 of [44]). In addition, we assume that for $r \rightarrow+\infty$, there is an asymptotic stationary solution denoted as $\left\{\left(\boldsymbol{U}_{\boldsymbol{x}}^{\text {stat }}(r), \boldsymbol{V}_{\boldsymbol{x}}^{\text {stat }}(r)\right), r \in \mathbb{R}^{+}\right\}$. Let $P_{S}\left(\mathrm{~d} \boldsymbol{u}, \mathrm{d} \boldsymbol{v} ; \boldsymbol{\lambda}_{\boldsymbol{x}}\right)$ be the invariant measure associated with the aforementioned unique stationary solution which can be obtained by taking $P_{\boldsymbol{U}_{\boldsymbol{x}}^{0}}, \boldsymbol{V}_{\boldsymbol{x}}^{0}(\mathrm{~d} \boldsymbol{u}, \mathrm{d} \boldsymbol{v} ; \boldsymbol{x})=P_{S}\left(\mathrm{~d} \boldsymbol{u}, \mathrm{d} \boldsymbol{v} ; \boldsymbol{\lambda}_{\boldsymbol{x}}\right)$. It follows that whenever the probability distribution $P_{\boldsymbol{U}_{\boldsymbol{x}}^{0}}, \boldsymbol{V}_{\boldsymbol{x}}^{\mathrm{o}}(\mathrm{d} \boldsymbol{u}, \mathrm{d} \boldsymbol{v} ; \boldsymbol{x})$ is different from the invariant measure $P_{S}\left(\mathrm{~d} \boldsymbol{u}, \mathrm{d} \boldsymbol{v} ; \boldsymbol{\lambda}_{\boldsymbol{x}}\right)$, the stochastic process $\left\{\left(\boldsymbol{U}_{\boldsymbol{x}}(r), \boldsymbol{V}_{\boldsymbol{x}}(r)\right), r \in \mathbb{R}^{+}\right\}$asymptotically converges in probability to the stationary process $\left\{\left(\boldsymbol{U}_{\boldsymbol{x}}^{\text {stat }}(r), \boldsymbol{V}_{\boldsymbol{x}}^{\text {stat }}(r)\right), r \in \mathbb{R}^{+}\right\}$as $r \rightarrow+\infty$. Note that the dissipative term generated by the family $\left\{f_{\boldsymbol{x}}^{0}\right\}_{\boldsymbol{x} \in \Omega}$ allows one to shorten the transient regime while solving the ISDE. In practice, the value of this field must be deduced beforehand from a parametric analysis on the rate of convergence towards the stationary solution (see $\S 3$ for an illustration). Let us introduce the probability density function $(\boldsymbol{u}, \boldsymbol{v}) \mapsto \rho_{S}\left(\boldsymbol{u}, \boldsymbol{v} ; \boldsymbol{\lambda}_{\boldsymbol{x}}\right)$ (with respect to $\mathrm{d} \boldsymbol{u} \mathrm{d} \boldsymbol{v}$ ) such that:

$$
P_{S}\left(\mathrm{~d} \boldsymbol{u}, \mathrm{d} \boldsymbol{v} ; \boldsymbol{\lambda}_{\boldsymbol{x}}\right)=\rho_{S}\left(\boldsymbol{u}, \boldsymbol{v} ; \boldsymbol{\lambda}_{\boldsymbol{x}}\right) \mathrm{d} \boldsymbol{u} \mathrm{d} \boldsymbol{v} .
$$


It can be shown that $\rho_{S}$ is a solution of a steady-state Fokker-Planck equation (FKPE; see [44] and the references therein for a survey, as well as [43] for illustrative examples in the case of nonlinear Hamiltonian dynamical systems) which explicitly depends on the potential function (see Eq. (21) of [48]) and which admits a unique solution (that can be related to the unique invariant measure of the ISDE), provided that $\Phi$ satisfies the following properties [48]:

$$
\begin{gathered}
\boldsymbol{u} \mapsto \Phi\left(\boldsymbol{u} ; \boldsymbol{\lambda}_{\boldsymbol{x}}\right) \text { is continuous on } \mathbb{R}^{N}, \\
\boldsymbol{u} \mapsto\left\|\nabla_{\boldsymbol{u}} \Phi\left(\boldsymbol{u} ; \boldsymbol{\lambda}_{\boldsymbol{x}}\right)\right\|_{\mathbb{R}^{N}} \text { is a locally bounded function on } \mathbb{R}^{N}, \\
\inf _{\|\boldsymbol{u}\|_{\mathbb{R}^{N}}>R} \Phi\left(\boldsymbol{u} ; \boldsymbol{\lambda}_{\boldsymbol{x}}\right) \rightarrow+\infty \text { as } R \rightarrow+\infty, \\
\inf _{\boldsymbol{u} \in \mathbb{R}^{N}} \Phi\left(\boldsymbol{u} ; \boldsymbol{\lambda}_{\boldsymbol{x}}\right)=\Phi^{\min }, \quad \Phi^{\min } \in \mathbb{R}, \\
\int_{\mathbb{R}^{N}}\left\|\nabla_{\boldsymbol{u}} \Phi\left(\boldsymbol{u} ; \boldsymbol{\lambda}_{\boldsymbol{x}}\right)\right\|_{\mathbb{R}^{N} p_{\boldsymbol{\lambda}_{\boldsymbol{x}}}(\boldsymbol{u} ; \boldsymbol{x}) \mathrm{d} \boldsymbol{u}<+\infty .}
\end{gathered}
$$

It is worth stressing that these properties must be checked carefully in practice, taking into account that the Lagrange multipliers belong to a given admissible set $\mathbb{D}_{\boldsymbol{\lambda}_{\boldsymbol{x}}}$. Consequently, the associated FKPE admits a unique solution, which implies the existence and uniqueness of the invariant measure $P_{S}$ associated with the ISDE defined by Eq. (2.41) [44]. Furthermore, one has

$$
\forall \boldsymbol{u} \in \mathbb{R}^{N}, p_{\boldsymbol{\lambda}_{\boldsymbol{x}}}(\boldsymbol{u} ; \boldsymbol{x})=\int_{\mathbb{R}^{N}} \rho_{S}\left(\boldsymbol{u}, \boldsymbol{v} ; \boldsymbol{\lambda}_{\boldsymbol{x}}\right) \mathrm{d} \boldsymbol{v}
$$

and

$$
\lim _{r \rightarrow+\infty} \boldsymbol{U}_{\boldsymbol{x}}(r)=\boldsymbol{Z}_{\boldsymbol{\lambda}_{\boldsymbol{x}}}
$$

in probability distribution [48]. From Eq. (2.40) and Eq. (2.49), it is seen that the above construction amounts to define the random field $\{\boldsymbol{G}(\boldsymbol{x}), \boldsymbol{x} \in \Omega\}$ as

$$
\boldsymbol{G}(\boldsymbol{x})=\mathcal{T}\left(\left\{\boldsymbol{W}_{\boldsymbol{x}}(r), r \geqslant 0\right\}, \boldsymbol{x}\right) \quad \forall \boldsymbol{x} \in \Omega,
$$

where $\mathcal{T}$ is a measurable nonlinear operator.

2.4.4. Discretization scheme. In this work, the ISDE is discretized by using a Störmer-Verlet algorithm. Such a scheme is well suited and efficient for Hamiltonian dynamical systems [58] (see [10] [22] for reviews about this scheme in the deterministic framework; see [7] and the references therein for the stochastic case; see [27] and [54] for general surveys on discretization schemes for stochastic differential equations, among others). Such a choice is motivated by the fact that we are considering the perturbation (by a dissipative term) of a Hamiltonian system. For $k=1, \ldots, M-1$, we let $\boldsymbol{U}_{\boldsymbol{x}}^{k}=\boldsymbol{U}_{\boldsymbol{x}}\left(r_{k}\right), \boldsymbol{V}_{\boldsymbol{x}}^{k}=\boldsymbol{V}_{\boldsymbol{x}}\left(r_{k}\right)$ and $r_{k}=(k-1) \Delta r$, where $\Delta r$ denotes the sampling step. For $k=1, \ldots, M-1$, the scheme writes

$$
\left\{\begin{aligned}
\boldsymbol{U}_{\boldsymbol{x}}^{k+1 / 2} & =\boldsymbol{U}_{\boldsymbol{x}}^{k}+\frac{\Delta r}{2} \boldsymbol{V}_{\boldsymbol{x}}^{k} \\
\boldsymbol{V}_{\boldsymbol{x}}^{k+1} & =\frac{1-a}{1+a} \boldsymbol{V}_{\boldsymbol{x}}^{k}+\frac{\Delta r}{1+a} \boldsymbol{L}_{\boldsymbol{x}}^{k+1 / 2}+\sqrt{f_{\boldsymbol{x}}^{0}} \Delta \boldsymbol{W}_{\boldsymbol{x}}^{k+1} \\
\boldsymbol{U}_{\boldsymbol{x}}^{k+1} & =\boldsymbol{U}_{\boldsymbol{x}}^{k+1 / 2}+\frac{\Delta r}{2} \boldsymbol{V}_{\boldsymbol{x}}^{k+1}
\end{aligned}\right.
$$


where $a=f_{\boldsymbol{x}}^{0} \Delta r / 4, \Delta \boldsymbol{W}_{\boldsymbol{x}}^{k+1}=\boldsymbol{W}_{\boldsymbol{x}}\left(r_{k+1}\right)-\boldsymbol{W}_{\boldsymbol{x}}\left(r_{k}\right)$ is the increment of the Wiener process between $r_{k+1}$ and $r_{k}, \boldsymbol{U}_{\boldsymbol{x}}^{1}=\boldsymbol{u}_{\boldsymbol{x}}^{0}$ and $\boldsymbol{V}_{\boldsymbol{x}}^{1}=\boldsymbol{v}_{\boldsymbol{x}}^{0}\left(\boldsymbol{u}_{\boldsymbol{x}}^{0}\right.$ and $\boldsymbol{v}_{\boldsymbol{x}}^{0}$ being arbitrary deterministic vectors) for all $\boldsymbol{x}$ in $\Omega$. The $\mathbb{R}^{N}$-valued random variable $\boldsymbol{L}_{\boldsymbol{x}}^{k}$ is defined as

$$
\left(\boldsymbol{L}_{\boldsymbol{x}}^{k}\right)_{j}=-\left\{\frac{\partial \Phi\left(\boldsymbol{u} ; \boldsymbol{\lambda}_{\boldsymbol{x}}\right)}{\partial u_{j}}\right\}_{\boldsymbol{u}=\boldsymbol{U}_{\boldsymbol{x}}^{k}}, j=1, \ldots, N,
$$

where the partial derivatives can analytically be evaluated using Eq. (2.37). Two very important properties of this scheme are worth mentioning. First, the algorithm turns out to be explicit (and thus, conditionally stable) in the present case, as the random variable $\boldsymbol{L}_{\boldsymbol{x}}^{k}$ does not depend on any time-evaluation of the velocity $\boldsymbol{V}_{\boldsymbol{x}}$. In practice, the equality (in probability distribution) stated by Eq. (2.49) can be written as

$$
\boldsymbol{G}(\boldsymbol{x})=\lim _{\Delta r \downarrow 0}\left(\lim _{k \rightarrow+\infty} \boldsymbol{U}_{\boldsymbol{x}}\left(r_{k}\right)\right)
$$

so that the convergence towards the target stationary solution must be investigated as $\Delta r \downarrow 0$, either in the sense of probability distribution (whenever an unbiased reference solution for the marginal distribution is available, for instance) or by comparing the convergence of some statistical moments for a given mapping acting on $\boldsymbol{U}_{\boldsymbol{x}}$ (see $\S 3$ ). Secondly, the proposed method allows for the preservation of the statistical dependence between the components of random vector $\boldsymbol{M}(\boldsymbol{x})$, regardless of the probabilistic dimension $N$.

From a computational point of view, solving the ISDE at point $\boldsymbol{x}$ requires the generation of independent copies of a $\mathbb{R}^{N}$-valued centered Gaussian random variable with covariance $\Delta r\left[I_{N}\right]$. Taking into account Eqs. $(2.36-2.35)$, it is seen that the cornerstone of the algorithm consists in defining the aforementionned copies as the independent copies of a second-order normalized Gaussian random variable $\boldsymbol{\Xi}_{\boldsymbol{x}}:=$ $\boldsymbol{\Xi}(\boldsymbol{x})$ (recall that $\boldsymbol{x}$ is fixed in $\Omega$ ). In other words, the Gaussian increment $\Delta \boldsymbol{W}_{\boldsymbol{x}}^{k+1}$ is set to be

$$
\Delta \boldsymbol{W}_{\boldsymbol{x}}^{k+1}=\boldsymbol{W}_{\boldsymbol{x}}\left(r_{k+1}\right)-\boldsymbol{W}_{\boldsymbol{x}}\left(r_{k}\right):=\sqrt{\Delta r} \boldsymbol{\Xi}_{\boldsymbol{x}}^{k+1} \quad \forall k \geqslant 1,
$$

where $\boldsymbol{\Xi}_{\boldsymbol{x}}^{k+1}$ denotes the $(k+1)$-th independent copy of random variable $\boldsymbol{\Xi}_{\boldsymbol{x}}$ (which is associated with the $(k+1)$-th independent copy of the random field $\left\{\boldsymbol{\Xi}(\boldsymbol{x}), \boldsymbol{x} \in \mathbb{R}^{d}\right\}$ defined in $\S 2.2)$. The random field $\{\boldsymbol{G}(\boldsymbol{x}), \boldsymbol{x} \in \Omega\}$ is then equivalently defined as

$$
\boldsymbol{G}(\boldsymbol{x})=\widetilde{\mathcal{T}}\left(\left\{\boldsymbol{\Xi}^{(k)}(\boldsymbol{x}), k \geqslant 1\right\}, \boldsymbol{x}\right) \quad \forall \boldsymbol{x} \in \Omega
$$

with $\widetilde{\mathcal{T}}$ a measurable operator. For all $\boldsymbol{x}$ in $\Omega$ and within the asymptotic regime corresponding to the stationary solution, such a strategy generates through the spatial correlations of the Gaussian random field $\left\{\boldsymbol{\Xi}(\boldsymbol{x}), \boldsymbol{x} \in \mathbb{R}^{d}\right\}$ the spatial dependencies for the random field $\{\boldsymbol{G}(\boldsymbol{x}), \boldsymbol{x} \in \Omega\}$ (and then, for $\{\mathcal{M}(\boldsymbol{x}), \boldsymbol{x} \in \Omega\}$ and $\{\boldsymbol{S}(\boldsymbol{x}), \boldsymbol{x} \in$ $\Omega\}$ ) that may be required while modeling the elasticity tensor random field (see $\S 3$ for numerical illustrations). It is worth pointing out that by proceeding this way, the random field $\{\boldsymbol{M}(\boldsymbol{x}), \boldsymbol{x} \in \Omega\}$ then inherits spatial dependencies (and thus, a correlation structure) which is a priori unknown and which depends on the choice of the correlation functions retained for the Gaussian stochastic germs. The proposed class of prior stochastic models therefore depends on a family of correlation functions which may be calibrated through an inverse identification, as indicated in $\S 2.2$. 
2.5. Prior stochastic representation for the random field $\{[C(x)], x \in$ $\Omega\}$. In order to obtain some flexibility with respect to the probabilistic modeling of material symmetries, we propose to decompose the random matrix $[\boldsymbol{C}(\boldsymbol{x})]$ as follows [19]:

$$
\forall \boldsymbol{x} \in \Omega, \quad[\boldsymbol{C}(\boldsymbol{x})]=[\boldsymbol{S}(\boldsymbol{x})][\boldsymbol{A}(\boldsymbol{x})][\boldsymbol{S}(\boldsymbol{x})]
$$

where

1. The random variables $[\boldsymbol{S}(\boldsymbol{x})]$ and $[\boldsymbol{A}(\boldsymbol{x})]$ are statistically independent.

2. $[\boldsymbol{S}(\boldsymbol{x})]$ is the unique positive-definite square root of the symmetric random matrix $[\mathcal{M}(\boldsymbol{x})]$, with $\{[\mathcal{M}(\boldsymbol{x})], \boldsymbol{x} \in \Omega\}$ the $\mathbb{M}_{n}^{\text {sym }}(\mathbb{R})$-valued random field defined in $\S 2.4$.

3. $\{[\boldsymbol{A}(\boldsymbol{x})], \boldsymbol{x} \in \Omega\}$ the random field (exhibiting anisotropic statistical fluctuations) defined in $\S 2.3$.

As $\{[\boldsymbol{S}(\boldsymbol{x})], \boldsymbol{x} \in \Omega\}$ and $\{[\boldsymbol{A}(\boldsymbol{x})], \boldsymbol{x} \in \Omega\}$ are independent random fields, it follows that the marginal p.d.f. $p_{[\boldsymbol{C}(\boldsymbol{x})]}$ of $[\boldsymbol{C}(\boldsymbol{x})]$ at point $\boldsymbol{x}$ is completely defined by the p.d.f. $p_{[\boldsymbol{S}(\boldsymbol{x})]}$ and $p_{[\boldsymbol{A}(\boldsymbol{x})]}$, or equivalently by the p.d.f. $p_{[\mathcal{M}(\boldsymbol{x})]}$ of $[\boldsymbol{M}(\boldsymbol{x})]$ and $p_{[\boldsymbol{A}(\boldsymbol{x})]}$. From the above set of properties, it can be easily deduced that:

- The random matrix $[\boldsymbol{C}(\boldsymbol{x})]$ is positive-definite a.s., with

$$
\mathbb{E}\{\log (\operatorname{det}([\boldsymbol{C}(\boldsymbol{x})]))\}=\nu(\boldsymbol{x})+\nu_{[\boldsymbol{A}]}(\boldsymbol{x}),|\nu(\boldsymbol{x})|+\left|\nu_{[\boldsymbol{A}]}(\boldsymbol{x})\right|<+\infty .
$$

- The mean function of random field $\{[\boldsymbol{C}(\boldsymbol{x})], \boldsymbol{x} \in \Omega\}$ is such that

$$
\forall \boldsymbol{x} \in \Omega, \quad \mathbb{E}\{[\boldsymbol{C}(\boldsymbol{x})]\}=[\underline{\mathcal{M}}(\boldsymbol{x})] .
$$

- $\{[\boldsymbol{C}(\boldsymbol{x})], \boldsymbol{x} \in \Omega\}$ and $\left\{[\boldsymbol{C}(\boldsymbol{x})]^{-1}, \boldsymbol{x} \in \Omega\right\}$ are second-order random fields,

$$
\forall \boldsymbol{x} \in \Omega, \quad \mathbb{E}\left\{\|[\boldsymbol{C}(\boldsymbol{x})]\|_{\mathrm{F}}^{2}\right\}<+\infty \quad \text { and } \quad \mathbb{E}\left\{\left\|[\boldsymbol{C}(\boldsymbol{x})]^{-1}\right\|_{\mathrm{F}}^{2}\right\}<+\infty .
$$

- The correlation structure of the random field $\{[\boldsymbol{C}(\boldsymbol{x})], \boldsymbol{x} \in \Omega\}$ is induced by the transformation of the correlation structures of the Gaussian stochastic germs by the nonlinear mappings $\mathcal{H}$ and $\widetilde{\mathcal{T}}$.

From Eq. (2.56), it can be inferred that the overall level of statistical fluctuations exhibited by the elasticity tensor random field $\{[\boldsymbol{C}(\boldsymbol{x})], \boldsymbol{x} \in \Omega\}$ depends on and increases together with the level of fluctuations exhibited by the random fields $\{[\boldsymbol{A}(\boldsymbol{x})], \boldsymbol{x} \in \Omega\}$ and $\{[\boldsymbol{M}(\boldsymbol{x})], \boldsymbol{x} \in \Omega\}$. Such a property also follows from Eq. (2.57), since the parameters $\delta_{[\boldsymbol{A}]}(\boldsymbol{x})$ and $\delta_{[M]}(\boldsymbol{x})$ are nonlinear functions of $\nu_{[\boldsymbol{A}]}(\boldsymbol{x})$ and $\nu(\boldsymbol{x})$ respectively (see [19] for numerical evidences). It should be noticed that by construction, setting $\delta_{[\boldsymbol{A}]}(\boldsymbol{x}) \rightarrow 0$ allows realizations of $[\boldsymbol{C}(\boldsymbol{x})]$ belonging a.s. to $\mathbb{M}_{n}^{\mathrm{sym}}(\mathbb{R})$ to be obtained (since this implies that $[\boldsymbol{A}(\boldsymbol{x})] \rightarrow\left[I_{n}\right]$ in probability; see [45]). Finally, note that the probabilistic model can handle a non-homogeneous system of first-order marginal probability distributions, so that it can be used for modeling mesoscopic fields associated with a non-stationary microstructure (e.g. functionally graded materials). In the next section, we exemplify the approach on the class of almost isotropic tensors.

3. Application to almost isotropic elasticity tensor random fields. For illustration purposes, we consider a one-dimensional domain $\Omega \subset \mathbb{R}$ and assume that the mean function, $\boldsymbol{x} \mapsto \delta_{[\boldsymbol{A}]}(\boldsymbol{x})$ and $\boldsymbol{x} \mapsto \nu(\boldsymbol{x})$ are constant over $\Omega$. Consequently, the first-order marginal p.d.f. of the auxiliary random fields do not depend on $\boldsymbol{x}$ either and any related subscript emphasizing spatial indexation is then dropped off from now on. Furthermore, we set $f_{\boldsymbol{x}}^{0}=f^{0}=9.5$ for all $\boldsymbol{x}$ in $\Omega$ and all the correlation lengths associated with $\{\boldsymbol{\xi}(\boldsymbol{x}), \boldsymbol{x} \in \Omega\}$ (resp. with $\{\boldsymbol{\Xi}(\boldsymbol{x}), \boldsymbol{x} \in \Omega\}$ ) are set to the same value $\mathcal{L}^{\text {ani }}$ (resp. $\left.\mathcal{L}^{\text {sym }}\right)$. 
3.1. Problem statement. We denote as $[J]$ and $[K]$ the matrix representations of the fourth-order symmetric tensors $\AA$ and $\mathbb{k}$, defined as $\AA_{i j k \ell}=(1 / 3) \delta_{i j} \delta_{k \ell}$ and $\mathbb{k}_{i j k \ell}=\left(\delta_{i k} \delta_{j \ell}+\delta_{i \ell} \delta_{j k}\right) / 2-\AA_{i j k \ell}$, constituting the classical basis of $\mathbb{M}_{n}^{\text {iso }}(\mathbb{R})$. In this application, we set $[\underline{\mathcal{M}}(\boldsymbol{x})]=3 \underline{M}_{1}[J]+2 \underline{M}_{2}[K]$, where the mean bulk and shear moduli are respectively given by $\underline{M}_{1}=1.5$ and $\underline{M}_{2}=1$ (both in [GPa]), whereas we take $\nu=-0.2$.

The random matrix $[\mathcal{G}(\boldsymbol{x})]$ can then be decomposed as:

$$
[\mathcal{G}(\boldsymbol{x})]=G_{1}(\boldsymbol{x})[J]+G_{2}(\boldsymbol{x})[K] \quad \forall \boldsymbol{x} \in \Omega .
$$

Note that

$$
[\mathcal{N}(\boldsymbol{x})]=\exp \left\{G_{1}(\boldsymbol{x})\right\}[J]+\exp \left\{G_{2}(\boldsymbol{x})\right\}[K] \quad \forall \boldsymbol{x} \in \Omega
$$

and then,

$$
[\boldsymbol{M}(\boldsymbol{x})]=3 \underline{M}_{1} \exp \left\{G_{1}(\boldsymbol{x})\right\}[J]+2 \underline{M}_{2} \exp \left\{G_{2}(\boldsymbol{x})\right\}[K] \quad \forall \boldsymbol{x} \in \Omega .
$$

Owing to the fact that $[J]$ and $[K]$ are commutating projectors (with $\operatorname{tr}([J])=1$ and $\operatorname{tr}([K])=5)$, it can be shown that Eq. (2.31) reduces to

$$
\begin{aligned}
p_{\boldsymbol{G}}(\boldsymbol{g}) & =c^{\boldsymbol{G}} \exp \left(-\lambda^{(1)} \exp \left\{g_{1}\right\}-\lambda g_{1}-5\left(\lambda^{(2)} \exp \left\{g_{2}\right\}+\lambda g_{2}\right)\right), \\
& :=p_{G_{1}}\left(g_{1}\right) \times p_{G_{2}}\left(g_{2}\right)
\end{aligned}
$$

with

$$
p_{G_{1}}\left(g_{1}\right)=c^{G_{1}} \exp \left(-\lambda^{(1)} \exp \left\{g_{1}\right\}-\lambda g_{1}\right) \quad \forall g_{1} \in \mathbb{R}
$$

and

$$
p_{G_{2}}\left(g_{2}\right)=c^{G_{2}} \exp \left(-5\left(\lambda^{(2)} \exp \left\{g_{2}\right\}+\lambda g_{2}\right)\right) \quad \forall g_{2} \in \mathbb{R},
$$

$c^{G_{1}}$ and $c^{G_{2}}$ being the two normalization constants. Eq. (3.4) shows that $\left\{G_{1}(\boldsymbol{x}), \boldsymbol{x} \in\right.$ $\Omega\}$ and $\left\{G_{2}(\boldsymbol{x}), \boldsymbol{x} \in \Omega\right\}$ are statistically independent random fields whose first-order p.d.f. depend in part on the same parameter $\lambda$. Note that this independence property stems from the second-order constraints that are integrated within the MaxEnt formulation and from the orthogonality of the tensor basis. In addition, the integrability of the above p.d.f. implies that $\lambda^{(1)}>0, \lambda^{(2)}>0$ and $\lambda<-1$. The gradient of the potential function is equal to:

$$
\forall \boldsymbol{u} \in \mathbb{R}^{2}, \quad \nabla_{\boldsymbol{u}} \Phi(\boldsymbol{u} ; \boldsymbol{\lambda})=\left(\lambda^{(1)} \exp \left\{u_{1}\right\}+\lambda, 5\left(\lambda^{(2)} \exp \left\{u_{2}\right\}+\lambda\right)\right) .
$$

The proof of existence for the stationary solution associated with such a potential function is provided in Appendix C. The constraints can then be written as:

$$
\begin{gathered}
\mathbb{E}\left\{\exp \left(G_{1}\right)\right\}=1, \\
\mathbb{E}\left\{\exp \left(G_{2}\right)\right\}=1, \\
\mathbb{E}\left\{G_{1}\right\}+5 \mathbb{E}\left\{G_{2}\right\}=\nu .
\end{gathered}
$$


In order to compute the Lagrange multipliers, we introduce the cost function $\boldsymbol{\lambda} \mapsto$ $\mathcal{J}(\boldsymbol{\lambda})$ (with $\left.\boldsymbol{\lambda}=\left(\lambda^{(1)}, \lambda^{(2)}, \lambda\right) \in \mathbb{D}_{\boldsymbol{\lambda}}\right)$ defined as:

$$
\mathcal{J}_{\alpha}(\boldsymbol{\lambda})=(1-\alpha) \frac{\|\widehat{\boldsymbol{e}}(\boldsymbol{\lambda})-\boldsymbol{e}\|^{2}}{\|\boldsymbol{e}\|^{2}}+\alpha \frac{(\widehat{\nu}(\boldsymbol{\lambda})-\nu)^{2}}{\nu^{2}} \quad \forall \boldsymbol{\lambda} \in \mathbb{D}_{\boldsymbol{\lambda}},
$$

where $0<\alpha<1$ and $\boldsymbol{e}=(1,1)$. In Eq. (3.11), $\widehat{\boldsymbol{e}}(\boldsymbol{\lambda})$ and $\widehat{\nu}(\boldsymbol{\lambda})$ are the following ergodic estimators related to the mathematical expectations involved in Eqs. (3.8$3.9)$ and Eq. (3.10) respectively:

$$
\begin{gathered}
\widehat{\boldsymbol{e}}(\boldsymbol{\lambda}) \simeq \frac{1}{M-M_{0}+1} \sum_{k=M_{0}}^{M}\left(\exp \left(U_{1}^{k}(\boldsymbol{\lambda})\right), \exp \left(U_{2}^{k}(\boldsymbol{\lambda})\right)\right) \\
\widehat{\nu}(\boldsymbol{\lambda}) \simeq \frac{1}{M-M_{0}+1} \sum_{k=M_{0}}^{M} U_{1}^{k}(\boldsymbol{\lambda})+5 U_{2}^{k}(\boldsymbol{\lambda})
\end{gathered}
$$

where $M_{0}$ denotes any iteration number within the stationary regime, $M$ is such that $M \gg M_{0}$ and the dependence of $\left\{\boldsymbol{U}^{k}\right\}_{k \geqslant 1}$ on $\boldsymbol{\lambda}$ has been made explicit. In practice, the values of $M_{0}$ and $M$ must be determined from a probabilistic convergence analysis (see below), whereas the value of parameter $\alpha$ must be tuned in order to balance the contribution of the mean constraint in the cost function.

\subsection{Numerical results.}

3.2.1. Convergence analysis and computation of the solution Lagrange multipliers. In this section, we address the computation of the Lagrange multipliers and denote by $\widetilde{\boldsymbol{\lambda}}=\left(\widetilde{\lambda}^{1}, \widetilde{\lambda}^{2}, \widetilde{\lambda}\right)$ the initial guess that is used within the optimization procedure. Matching the modes of the p.d.f. with the known mean values, it is found that the components of $\widetilde{\boldsymbol{\lambda}}$ may be such that

$$
\widetilde{\lambda}^{1}=\widetilde{\lambda}^{2}=-\tilde{\lambda}
$$

hence allowing for a parametric analysis in terms of a single parameter, say $\tilde{\lambda}$. For any value of $\widetilde{\lambda}$, the convergence towards the stationary solution of the ISDE must be characterized. In this work, such a convergence is inferred from the convergence of the ergodic estimator for the second-order moment $\mathbb{E}\left\{\|\boldsymbol{U}\|^{2}\right\}$ of $\|\boldsymbol{U}\|$. From a practical point of view, such a strategy amounts to analyse the convergence of the mapping $\mathrm{N}_{\text {iter }} \mapsto$ ConvMes $\left(\mathrm{N}_{\text {iter }}\right)$ defined as:

$$
\operatorname{ConvMes}\left(\mathrm{N}_{\text {iter }}\right)=\frac{1}{\mathrm{~N}_{\text {iter }}} \sum_{k=1}^{\mathrm{N}_{\text {iter }}}\left\|\boldsymbol{U}^{k}\right\|^{2} .
$$

Meanwhile, the convergence of the diffusion w.r.t. $\Delta r$ must be characterized (see Eq. (2.53)). Below, we perform such a convergence analysis by characterizing the convergence of the mapping $\mathrm{N}_{\text {iter }} \mapsto \operatorname{ErrConv}\left(\mathrm{N}_{\text {iter }}\right)$ given by

$$
\operatorname{ErrConv}\left(\mathrm{N}_{\text {iter }}\right)=\left|\frac{\operatorname{ConvMes}\left(\mathrm{N}_{\text {iter }}\right)-m_{2}^{\text {Ref }}}{m_{2}^{\text {Ref }}}\right|
$$

where $m_{2}^{\text {Ref }}$ is the reference value for the second-order moment of $\|\boldsymbol{U}\|$ which is obtained (for a given value of the Lagrange multipliers) using a standard explicit EulerMaruyama scheme [27] with $\Delta r=10^{-5}$ and $M=10^{9}$. The graphs of mappings 


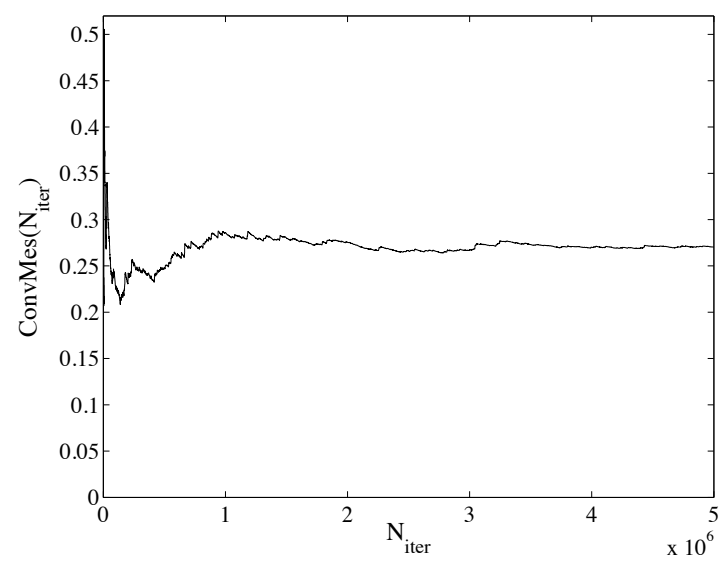

FIG. 3.1. Convergence towards the stationary solution of the ISDE: graph of $\mathrm{N}_{\mathrm{iter}} \mapsto$ ConvMes $\left(\mathrm{N}_{\text {iter }}\right)$ for $\Delta r=10^{-3}$.

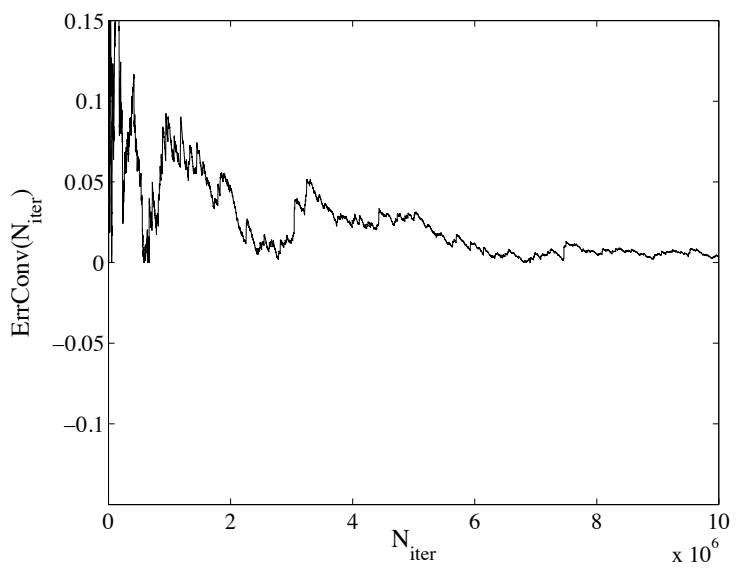

FIG. 3.2. Convergence of the ergodic estimator of $\|\boldsymbol{U}\|^{2}$ : graph of error function $\mathrm{N}_{\mathrm{iter}} \mapsto$ $\operatorname{ErrConv}\left(\mathrm{N}_{\text {iter }}\right)$ for $\Delta r=10^{-3}$.

$\mathrm{N}_{\text {iter }} \mapsto \operatorname{ConvMes}\left(\mathrm{N}_{\text {iter }}\right)$ and $\mathrm{N}_{\text {iter }} \mapsto \operatorname{ErrConv}\left(\mathrm{N}_{\text {iter }}\right)$ are displayed in Figs. 3.1 and 3.2 for $\widetilde{\lambda}=5$ and $\Delta r=10^{-3}$. It is seen that the stationary regime is reached for $M_{0} \geqslant 10^{6}$, whereas a good convergence of the diffusion is obtained for $\Delta r=10^{-3}$ (with a relative error for the second-order moment of about $0.3 \%$ for $M=10^{7}$ ). Note that this value of $\widetilde{\lambda}$ has been deduced from a preliminary optimization run with $M_{0}=10^{6}$ and $M=5 \times 10^{6}$. We now set $\Delta r=10^{-3}, M_{0}=10^{6}$ and $M=10^{7}$ for subsequent analysis. The convergence of the active-set optimization algorithm for $\widetilde{\lambda}=5$ is shown in Fig. 3.3 in semilog scale for $\alpha=0.6$. It is seen that the algorithm converges very fast (the computation time being about 355 seconds on a $2.8 \mathrm{GHz}$ single-core processor). The solution Lagrange multipliers are found to be:

$$
\lambda^{1}=5.0924, \lambda^{2}=5.0697, \lambda=-5.0712 .
$$




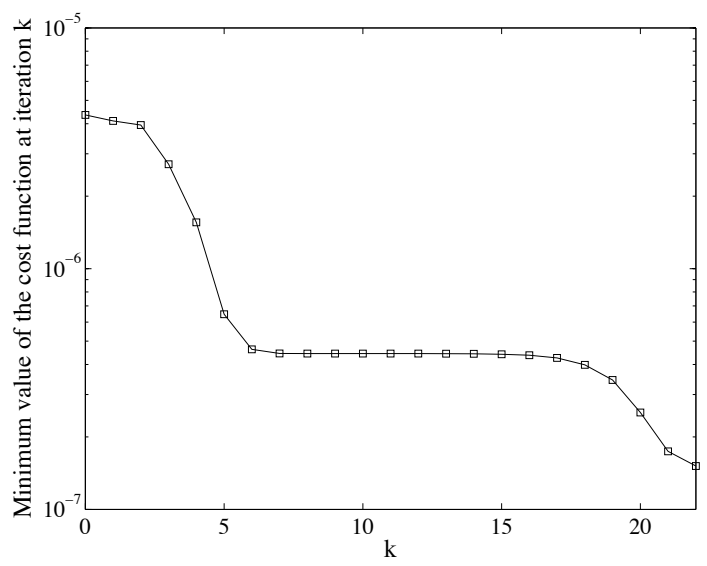

FIG. 3.3. Convergence of the optimization algorithm for the computation of the Lagrange multipliers.

The associated value of the cost function is $1.51 \times 10^{-7}$, corresponding to $\widehat{e}=$ $(1.0002,1.0008)$ and $\widehat{\nu}=-0.2$. The p.d.f. of $G_{1}$ and $G_{2}$ are shown in Fig. 3.4, where the densities have been obtained by using a classical kernel estimator and 10000 independent realizations.

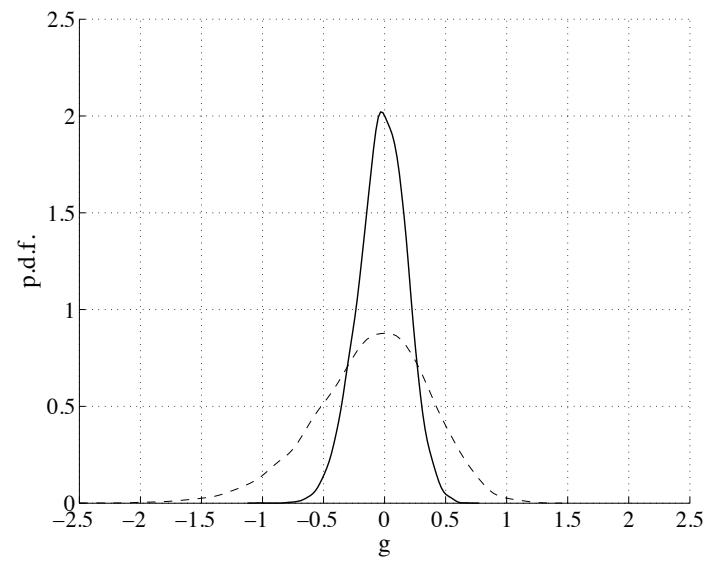
line).

FIG. 3.4. Plot of the marginal p.d.f.: graphs of $g \mapsto p_{G_{1}}(g)$ (dashed line) and $g \mapsto p_{G_{2}}(g)$ (solid

3.2.2. Random field application. In this section, we finally provide a few results that illustrate the random field case. To this aim, we set $\Omega=] 0,100[$ (in $[\mathrm{mm}]$ ) and the spatial correlation lengths are chosen as $\mathcal{L}^{\text {ani }}=\mathcal{L}^{\text {sym }}=20[\mathrm{~mm}]$. The marginal p.d.f. are those calibrated in the previous section. A few samples of the normalized random fields $\left\{G_{1}(x), x \in \Omega\right\}$ and $\left\{G_{2}(x), x \in \Omega\right\}$ are shown in Fig. 3.5. Fig. 3.6 shows the mean functions $x \mapsto \mathbb{E}\left\{G_{1}(x)\right\}, x \mapsto \mathbb{E}\left\{G_{2}(x)\right\}, x \mapsto \mathbb{E}\left\{C_{11}(x)\right\}$ and $x \mapsto \mathbb{E}\left\{C_{44}(x)\right\}$ (which have been estimated by using 1000 independent realizations). This figure illustrates in part the fact that both $\{\boldsymbol{G}(x), x \in \Omega\}$ and $\{[\boldsymbol{C}(x)], x \in \Omega\}$ 
can be seen as the restrictions to $\Omega$ of stationary random fields, since the mean value, $\nu$ (for the random field with values in $\mathbb{M}_{n}^{\text {iso }}(\mathbb{R})$ ) and $\delta$ (for the anisotropic germ) do not depend on $x$. Let Corr : $\mathbb{R} \rightarrow \mathbb{R}$ be the correlation function associated with the
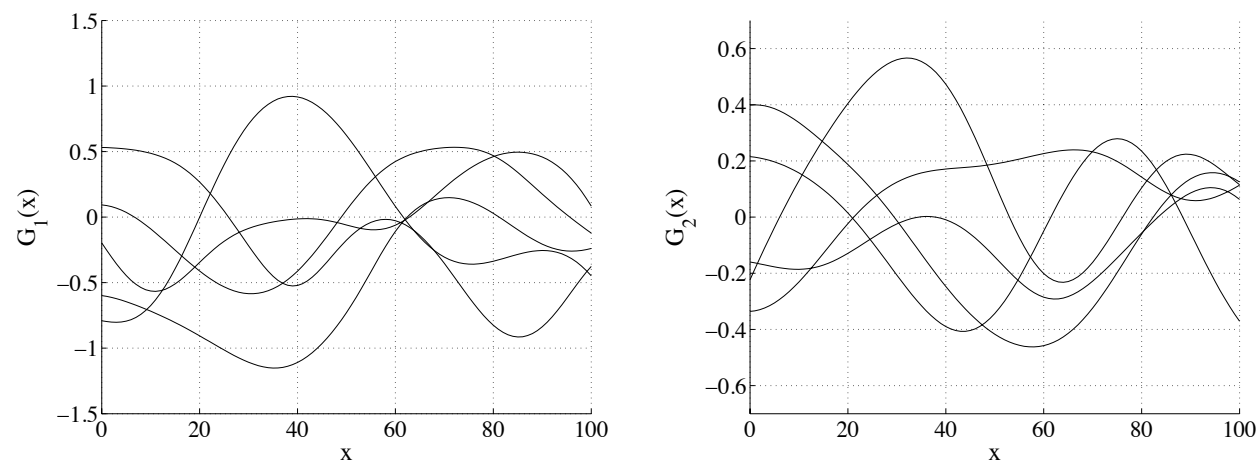

FiG. 3.5. Samples of random fields $\left\{G_{1}(x), x \in \Omega\right\}$ (left) and $\left\{G_{2}(x), x \in \Omega\right\}$ (right).
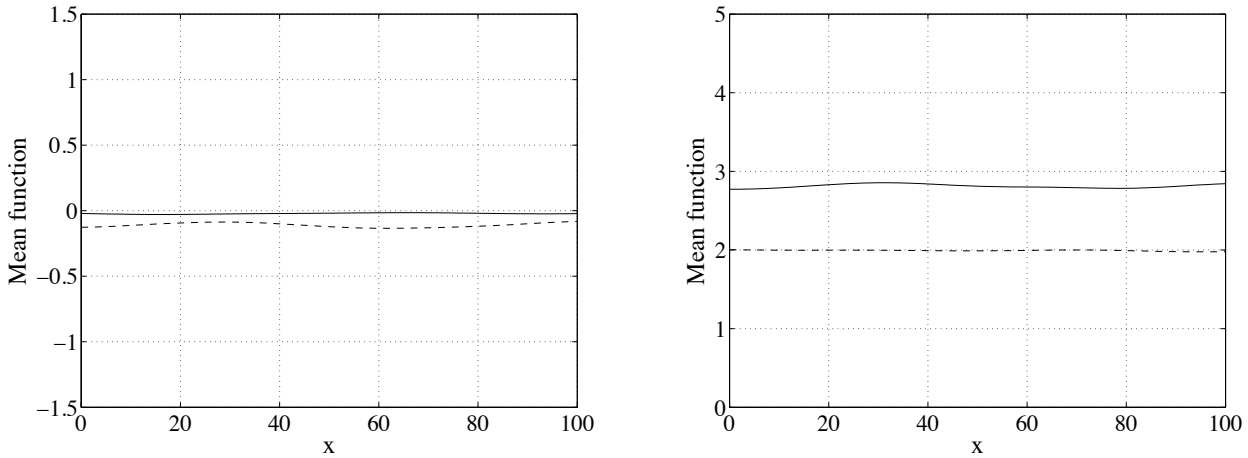

FIG. 3.6. Plot of mean functions. Left: $x \mapsto \mathbb{E}\left\{G_{1}(x)\right\}$ (dashed line) and $x \mapsto \mathbb{E}\left\{G_{2}(x)\right\}$ (solid line). Right: $x \mapsto \mathbb{E}\left\{C_{11}(x)\right\}$ (solid line) and $x \mapsto \mathbb{E}\left\{C_{44}(x)\right\}$ (dashed line).

elasticity tensor random field and defined as:

$$
\forall x \in \mathbb{R}, \quad \operatorname{Corr}(x)=\frac{\operatorname{tr} \mathbb{E}\left\{\left(\left[\boldsymbol{C}\left(x_{0}+x\right)\right]-[\underline{\mathcal{M}}]\right)\left(\left[\boldsymbol{C}\left(x_{0}\right)\right]-[\underline{\mathcal{M}}]\right)\right\}}{\mathbb{E}\left\{\left\|\left[\boldsymbol{C}\left(x_{0}\right)\right]-[\underline{\mathcal{M}}]\right\|_{\mathrm{F}}^{2}\right\}},
$$

where $x_{0}$ is any point in $\Omega$. Fig. 3.7 displays the plot of $x \mapsto \operatorname{Corr}(x)$. Other graphs of function Corr obtained for different input correlation functions are reported in Appendix D. In all cases, it is seen that the overall shape of the input correlation function is preserved, whereas a slight modification (typically of less than 10\%) of the correlation length is observed. Note that the same conclusion holds for the correlation functions associated with the components of the elasticity tensor random field. Finally, some joint p.d.f. for the components $(1,1)$ and $(4,4)$ of the random elasticity tensor at some points of the domain $\Omega$ are shown in Figs. 3.8, 3.9 and 3.10.

4. Conclusion. We have addressed the construction of a class of generalized non-parametric probabilistic models for matrix-valued non-Gaussian random fields which exhibit statistical fluctuations around a subset of the set of real symmetric 


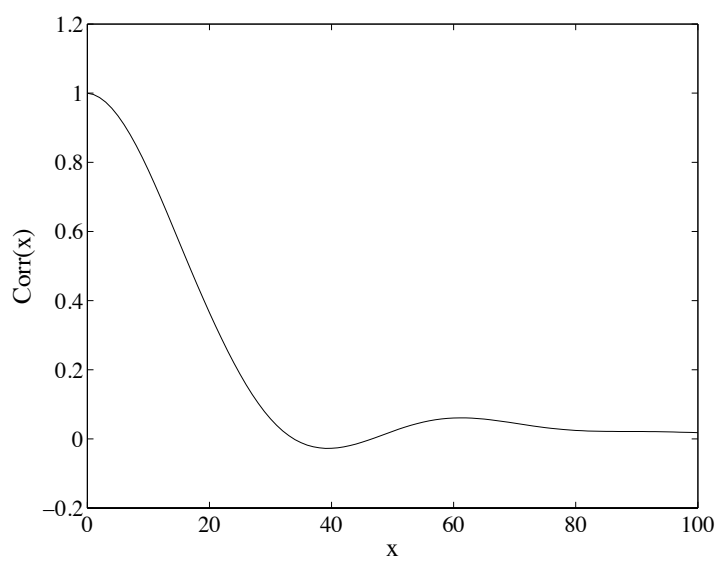

FIG. 3.7. Correlation function of the elasticity tensor random field: plot of $x \mapsto \operatorname{Corr}(x)$.

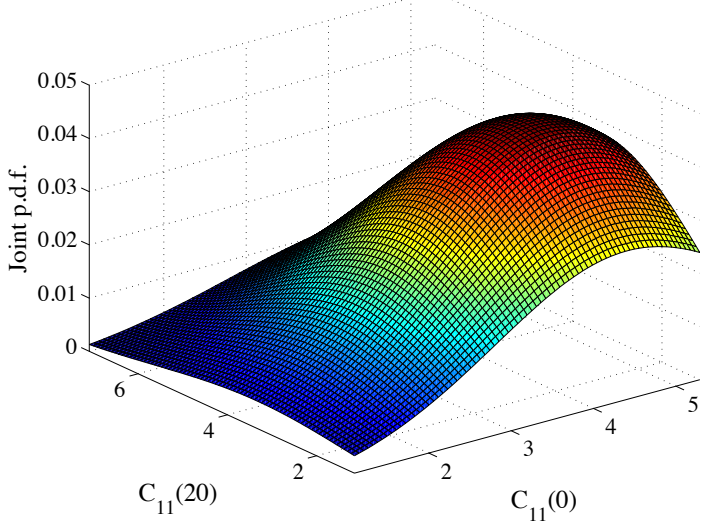

FIG. 3.8. Plot of the joint p.d.f. between $C_{11}(0)$ and $C_{11}(20)$.

positive-definite matrices presenting sparsity and invariance with respect to given orthogonal transformations. This issue naturally arises in multiscale analysis, where the mesoscale modeling of heterogeneous microstructures typically requires taking heed of both randomness and material symmetry constraints.

First, we have presented a methodology relying on the framework of information theory and defined a particular algebraic form for the random field. This representation involves two matrix-valued stochastic germs and offers some flexibility for forward simulations and inverse identification by uncoupling the levels of statistical fluctuations exhibited by the random field and by an associated stochastic anisotropy measure. Subsequently, we have proposed a novel stochastic modeling and an associated numerical strategy for the simulation of the random field. The approach consists in solving a family of Itô stochastic differential equations, indexed by the domain over which the realizations have to be simulated. The proposed algorithm allows for the preservation of the statistical dependence, at no additional computational cost, as the probabilistic dimension increases. A Störmer-Verlet discretization scheme is further 


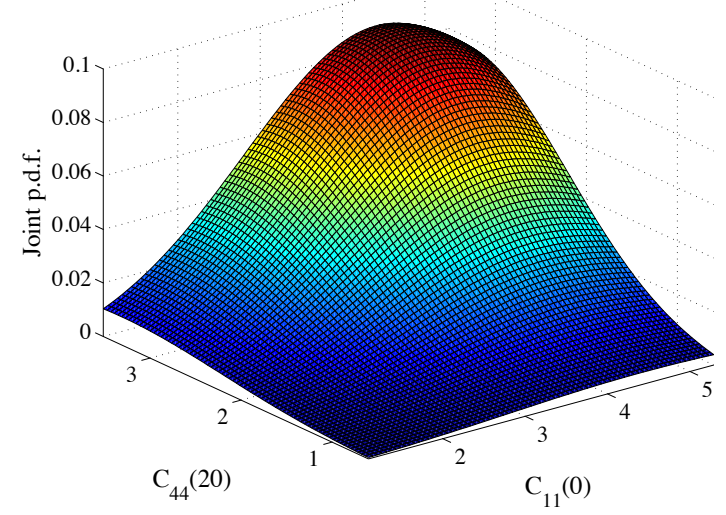

FIG. 3.9. Plot of the joint p.d.f. between $C_{11}(0)$ and $C_{44}(20)$.

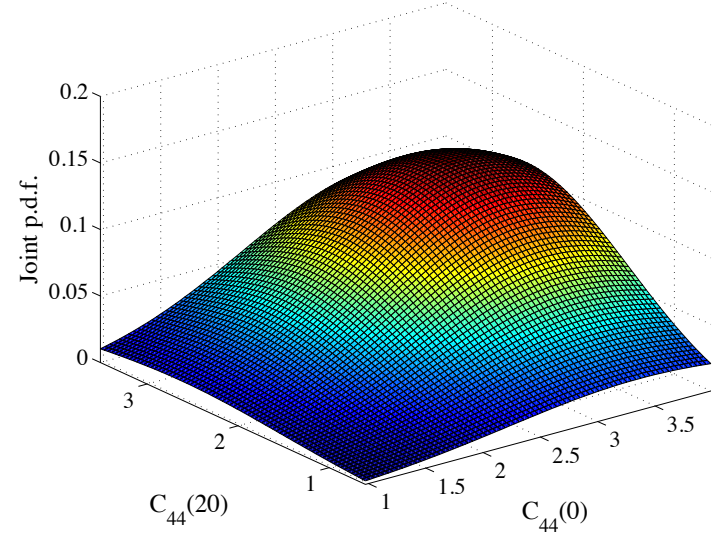

FIG. 3.10. Plot of the joint p.d.f. between $C_{44}(0)$ and $C_{44}(20)$.

formulated. This scheme is very efficient and turns out to converge much faster than an explicit euler scheme for instance. We then exemplified the approach and some related technicalities in the case of an elasticity tensor random field exhibiting some fluctuations around the class of isotropic tensors. In particular, it is shown that the model is able to generate correlation functions that are typically exhibited by elasticity tensor random fields (e.g. exponential-type and sinc-type correlation functions).

The approach and algorithm are both general and can be readily applied to the modeling of other non-Gaussian tensor-valued random fields exhibiting the same kind of symmetry properties, provided that a deterministic basis can span the associated subset of $\mathbb{M}_{n}^{+}(\mathbb{R})$.

Acknowledgements. The financial support of the French National Research Agency (Agence Nationale de la Recherche) is gratefully acknowledged. The work of J. Guilleminot is supported by ANR grants ANR-2010-BLAN-0904 and ANR-12-JS090001-01. The work of C. Soize is supported by ANR grant ANR-2010-BLAN-0904. 
Appendix A. Proof of proposition 2.1. In order to derive the proof for the proposition 2.1, we might distinguish two groups of material symmetries, namely the one where $\mathbb{M}_{n}^{\text {sym }}(\mathbb{R})$ is spanned by commutating projectors and for which closed-form expressions can be obtained (this is the case of the isotropic and cubic symmetries), and the one gathering the other symmetry classes (apart from the triclinic case). For each group, we provide a proof for a specific class which can be generalized, in a straightforward manner, to all classes of the same group ${ }^{2}$.

Let us consider the first group and address the case of the isotropic symmetry. Any matrix $[N] \in \mathbb{M}_{n}^{\text {iso }}(\mathbb{R})$ can then be written as

$$
[N]=N_{1}[J]+N_{2}[K]
$$

with $N_{1}>0, N_{2}>0$ (in a mechanical context, $N_{1} / 3$ and $N_{2} / 2$ correspond to the bulk and shear moduli, respectively) and where the matrices $[J]$ and $[K]$ have been defined in $\S 3.1$. Let $[A]$ be any symmetric matrix spanned by $[J]$ and $[K]:[A]=A_{1}[J]+$ $A_{2}[K]$, with $\left(A_{1}, A_{2}\right) \in \mathbb{R}^{2}$. Since $[J]$ and $[K]$ are commutating orthogonal projectors, it can be deduced that: $\operatorname{expm}([A])=\exp \left\{A_{1}\right\}[J]+\exp \left\{A_{2}\right\}[K]$. Consequently, and since $\left(N_{1}, N_{2}\right) \in \mathbb{R}_{*}^{+2}$ by definition, there exists a unique symmetric matrix $[G]$, defined as

$$
[G]=\log \left\{N_{1}\right\}[J]+\log \left\{N_{2}\right\}[K],
$$

such that $[N]=\operatorname{expm}([G])$.

Moving on to the other group, let us consider for instance the case of transversely isotropic elasticity matrices. We denote by $\boldsymbol{n}$ the unit vector normal to the plane of isotropy. Let $[p]$ and $[q]$ be the two second-rank (idempotent) symmetric tensors defined by $[p]=\boldsymbol{n} \otimes \boldsymbol{n}$ and $[q]=[I]-[p]$, with $[I]_{i j}=\delta_{i j}$. The basis $\left.\left\{\left[E_{\text {t.i. }}{ }^{(i)}\right]\right\}_{i=1}^{i=5}\right\}$ of $\mathbb{M}_{n}^{\text {t.i. }}(\mathbb{R})$ then corresponds to the Kelvin matrix representation of the following tensor basis:

$$
\begin{gathered}
\mathbb{e}^{(1)}=[p] \otimes[p], \mathbb{e}^{(2)}=\frac{1}{2}[q] \otimes[q], \mathbb{e}^{(3)}=\frac{1}{\sqrt{2}}([p] \otimes[q]+[q] \otimes[p]), \\
\mathbb{e}^{(4)}=[q] \underline{\otimes}[q]-\mathbb{e}^{(2)}, \mathbb{e}^{(5)}=\stackrel{\AA}{-} \mathbb{e}^{(1)}-\mathbb{e}^{(2)}-\mathbb{e}^{(4)},
\end{gathered}
$$

where $₫$ is the fourth-rank symmetric identity tensor given by $2 \AA_{i j k \ell}^{\circ}=\delta_{i k} \delta_{j \ell}+\delta_{i \ell} \delta_{j k}$. Any matrix $[N] \in \mathbb{M}_{n}^{\text {t.i. }}(\mathbb{R})$ can then be expanded as

$$
[N]=N_{1}\left[E_{\text {t.i. }}{ }^{(1)}\right]+N_{2}\left[E_{\text {t.i. }}{ }^{(2)}\right]+N_{3}\left[E_{\text {t.i. }}{ }^{(3)}\right]+N_{4}\left[E_{\text {t.i. }}{ }^{(4)}\right]+N_{5}\left[E_{\text {t.i. }}{ }^{(5)}\right],
$$

where the coefficients $N_{1}, \ldots, N_{5}$ belong to a subspace of $\mathbb{R}^{5}$ such that $[N]$ is positivedefinite. Eq. (A.3) can be rewritten in a symbolic form as

$$
[N]=\left\{\left[N_{123}\right], N_{4}, N_{5}\right\},
$$

in which $N_{4}>0, N_{5}>0$ and $\left[N_{123}\right]$ is the positive-definite $(2 \times 2)$ real matrix given by

$$
\left[N_{123}\right]=\left(\begin{array}{ll}
N_{1} & N_{3} \\
N_{3} & N_{2}
\end{array}\right)
$$

\footnotetext{
${ }^{2}$ There is a restriction for the monoclinic system, for which the proof holds provided that the system is defined with respect to three mutually orthogonal vectors. However, this does not appear as a severe limitation, since engineered or biological materials are unlikely to exhibit such a material symmetry property.
} 
The representation defined by Eq. (A.4) allows for simple algebraic calculations in $\mathbb{M}_{n}^{\text {t.i. }}(\mathbb{R})$, as shown in [59]. Let $[A]$ be any symmetric matrix spanned by $\left.\left\{\left[E_{\text {t.i. }}{ }^{(i)}\right]\right\}_{i=1}^{i=5}\right\}$ :

$$
[A]=A_{1}\left[E_{\text {t.i. }}{ }^{(1)}\right]+A_{2}\left[E_{\text {t.i. }}{ }^{(2)}\right]+A_{3}\left[E_{\text {t.i. }}{ }^{(3)}\right]+A_{4}\left[E_{\text {t.i. }}{ }^{(4)}\right]+A_{5}\left[E_{\text {t.i. }}{ }^{(5)}\right],
$$

in which $\left(A_{1}, \ldots, A_{5}\right) \in \mathbb{R}^{5}$. Upon using the algebraic properties of the matrix basis, it is easily seen (with obvious notations) that:

$$
\operatorname{expm}([A])=\left\{\operatorname{expm}\left(\left[A_{123}\right]\right), \exp \left\{A_{4}\right\}, \exp \left\{A_{5}\right\}\right\} .
$$

Since $\left[N_{123}\right]>0, N_{4}>0$ and $N_{5}>0$ by construction, it can then be deduced that there exists a unique symmetric matrix $[G]$ such that $[N]=\operatorname{expm}[G]$ and which is defined, in the symbolic form introduced above, as:

$$
[G]=\left\{\operatorname{logm}\left(\left[N_{123}\right]\right), \log \left\{N_{4}\right\}, \log \left\{N_{5}\right\}\right\} .
$$

Appendix B. Sketch of proof for the integrability of $p_{[\mathcal{N}(x)]}$. Let $\boldsymbol{x}$ be fixed in $\Omega$. The p.d.f. $p_{[\mathcal{N}(\boldsymbol{x})]}$ of $[\mathcal{N}(\boldsymbol{x})]$ with respect to the measure $\widetilde{\mathrm{d}} N=$ $2^{n(n-1) / 4} \prod_{1 \leqslant i \leqslant j \leqslant n} \mathrm{~d} N_{i j}$ reads as:

$$
\begin{aligned}
p_{[\mathcal{N}(\boldsymbol{x})]}([N] ; \boldsymbol{x})= & \mathbb{1}_{\mathbb{M}_{n}^{+}(\mathbb{R})}([N]) c_{\boldsymbol{x}}^{N} \operatorname{det}([N])^{-\lambda_{\boldsymbol{x}}^{\text {sol }}-1} \exp \left(-\ll\left[\Lambda_{\boldsymbol{x}}^{\text {sol }}\right],[N] \gg\right) \\
& \times \prod_{i<j}\left|\frac{\log \left(\rho_{j}\right)-\log \left(\rho_{i}\right)}{\rho_{j}-\rho_{i}}\right|,
\end{aligned}
$$

where $\rho_{1}<\ldots<\rho_{n}$ are the strictly positive eigenvalues of $[N]$. For any matrix $[N]$ in $\mathbb{M}_{n}^{+}(\mathbb{R})$, we introduce the spectral decomposition $[N]=[\Phi][\boldsymbol{\rho}][\Phi]^{\mathrm{T}}$, where $[\boldsymbol{\rho}]$ is the diagonal matrix gathering the eigenvalues of $[N]$ and $[\Phi]$ is the orthogonal matrix of associated eigenvectors. Upon introducing the classical representation of the orthogonal matrix $[\Phi]$, namely

$$
[\Phi]=[\Phi(\alpha, \boldsymbol{r})]=\operatorname{expm}(\alpha[H(\boldsymbol{r})]),
$$

with $\alpha \geqslant 0$ and $[H(\boldsymbol{r})] \in \mathbb{M}_{n}(\mathbb{R})$ skew-symmetric such that $\|[H(\boldsymbol{r})]\|_{\mathrm{F}}^{2}=1$, it can be shown that [45]:

$$
\begin{aligned}
p_{[\mathcal{N}(\boldsymbol{x})]}([N] ; \boldsymbol{x}) \widetilde{\mathrm{d}} N= & p_{[\mathcal{N}(\boldsymbol{x})]}\left([\Phi(\alpha, \boldsymbol{r})][\boldsymbol{\rho}][\Phi(\alpha, \boldsymbol{r})]^{\mathrm{T}}\right) \\
& \times 2^{n(n-1) / 4} g(\alpha, \boldsymbol{r}) \prod_{i<j}\left|\rho_{j}-\rho_{i}\right| \mathrm{d} \boldsymbol{\rho} \mathrm{d} \alpha \mathrm{d} \boldsymbol{r},
\end{aligned}
$$

in which $(\alpha, \boldsymbol{r}) \mapsto g(\alpha, \boldsymbol{r})$ is a positive bounded function [32] and $(\rho, \alpha, \boldsymbol{r}) \in \mathbb{D}_{\rho} \times$ $\mathbb{D}_{\alpha} \times \mathbb{D}_{r}$. Owing to a proper definition of the admissible set of $\left[\Lambda_{\boldsymbol{x}}^{\text {sol }}\right]$ such that $\ll$ $\left[\Lambda_{\boldsymbol{x}}^{\text {sol }}\right],[\Phi(\alpha, \boldsymbol{r})] \operatorname{logm}[\boldsymbol{\rho}][\Phi(\alpha, \boldsymbol{r})]^{\mathrm{T}} \gg \geqslant 0$ and upon substituting Eq. (B.1) in Eq. (B.3), it can be deduced that $p_{[\mathcal{N}(\boldsymbol{x})]}$ is integrable if and only if

$$
\int_{\|\rho\|<\epsilon} \operatorname{det}([N])^{-\lambda_{x}^{s o l}-1} \prod_{i<j}\left|\log \left(\rho_{j}\right)-\log \left(\rho_{i}\right)\right| \mathrm{d} \rho<+\infty
$$

for any $\epsilon$, with $0<\epsilon \ll 1$. Let $\mathcal{I}\left(\boldsymbol{\rho} ; \lambda_{\boldsymbol{x}}^{\text {sol }}\right)$ be the integrand defined in Eq. (B.4):

$$
\mathcal{I}\left(\boldsymbol{\rho} ; \lambda_{\boldsymbol{x}}^{\text {sol }}\right)=\operatorname{det}([N])^{-\lambda_{\boldsymbol{x}}^{\text {sol }}-1} \prod_{i<j}\left|\log \left(\rho_{j}\right)-\log \left(\rho_{i}\right)\right| .
$$


Since $\operatorname{det}([N])=\prod_{i} \rho_{i}$ and $\rho_{i}<\rho_{j}$ for $i<j$, it follows that

$$
\mathcal{I}\left(\boldsymbol{\rho} ; \lambda_{\boldsymbol{x}}^{s o l}\right) \leqslant 2^{n(n-1) / 2}\left(\prod_{i} \rho_{i}\right)^{\beta} \prod_{2 \leqslant i \leqslant n}\left|\log \rho_{i}\right|^{i-1},
$$

in which $\beta=-\lambda_{x}^{\text {sol }}-1$ and use has been made of the inequality $|x-y| \leqslant|x|+|y|$ for $(x, y) \in \mathbb{R}^{2}$. It can be deduced that:

$$
\int_{\|\rho\|<\epsilon} \mathcal{I}\left(\boldsymbol{\rho} ; \lambda_{\boldsymbol{x}}^{\text {sol }}\right) \mathrm{d} \boldsymbol{\rho} \leqslant 2^{n(n-1) / 2} \int_{\|\rho\|<\epsilon} \rho_{1}^{\beta} \prod_{2 \leqslant i \leqslant n}\left|\rho_{i}^{\beta /(i-1)} \log \rho_{i}\right|^{i-1} \mathrm{~d} \boldsymbol{\rho} .
$$

Provided that $\beta>0$ (i.e. $\lambda_{\boldsymbol{x}}^{\text {sol }}<-1$ ), it is clear that the right hand side of Eq. (B.7) is finite, and so is the left-hand side of Eq. (B.4).

Appendix C. Proof of existence for the stationary solution associated the numerical application. We examine in this appendix the existence of the stationary solution for the potential function introduced in $\S 3.1$ :

$$
\forall \boldsymbol{u} \in \mathbb{R}^{2}, \quad \Phi(\boldsymbol{u} ; \boldsymbol{\lambda})=\lambda^{(1)} \exp \left\{u_{1}\right\}+\lambda u_{1}+5\left(\lambda^{(2)} \exp \left\{u_{2}\right\}+\lambda u_{2}\right) .
$$

Let us decompose $\Phi$ as

$$
\forall \boldsymbol{u} \in \mathbb{R}^{2}, \quad \Phi(\boldsymbol{u} ; \boldsymbol{\lambda}):=\Phi_{1}\left(u_{1} ; \lambda^{(1)}, \lambda\right)+\Phi_{2}\left(u_{2} ; \lambda^{(2)}, \lambda\right),
$$

with $\Phi_{1}\left(u_{1} ; \lambda^{(1)}, \lambda\right)=\lambda^{(1)} \exp \left\{u_{1}\right\}+\lambda u_{1}$ and $\Phi_{2}\left(u_{2} ; \lambda^{(2)}, \lambda\right)=5\left(\lambda^{(2)} \exp \left\{u_{2}\right\}+\lambda u_{2}\right)$. It follows that the two-dimensional ISDE consists of two uncoupled one-dimensional ISDE's. The existence and uniqueness of a stationary solution can then be proven by checking that either $\Phi_{1}$ or $\Phi_{2}$ satisfies the properties given by Eqs. (2.43-2.47) (since these potentials only differ by a multiplicative constant). Let us consider $\Phi_{1}$ and note first that $\lambda^{(1)}>0$ and $\lambda<0$. One has $\lim _{u_{1} \rightarrow+\infty} \inf \Phi_{1}\left(u_{1} ; \lambda^{(1)}, \lambda\right)=+\infty$. Furthermore, it is clear that $\nabla \Phi_{1}\left(u_{1} ; \lambda^{(1)}, \lambda\right)=\lambda^{(1)} \exp \left\{u_{1}\right\}+\lambda$ is locally bounded on $\mathbb{R}$, so that the conditions given by Eqs. $(2.43-2.45)$ are all trivially satisfied. One has $\inf _{u_{1} \in \mathbb{R}} \Phi_{1}\left(u_{1} ; \lambda^{(1)}, \lambda\right)=\Phi_{1}\left(\log \left\{-\lambda / \lambda^{(1)}\right\} ; \lambda^{(1)}, \lambda\right)$, which is well defined since $\lambda / \lambda^{(1)}<0$. Therefore, and since $-\lambda<+\infty$ and $-\lambda / \lambda^{(1)} \approx 1$ in practice (see $\S 3.2 .1$ ), one has:

$$
\inf _{u_{1} \in \mathbb{R}} \Phi_{1}\left(u_{1} ; \lambda^{(1)}, \lambda\right)=\lambda\left(\log \left\{-\lambda / \lambda^{(1)}\right\}-1\right)<+\infty .
$$

Hence, the condition stated by Eq. (2.46) is also satisfied. Finally, one has

$$
\int_{\mathbb{R}}\left|\nabla \Phi_{1}\left(u_{1} ; \lambda^{(1)}, \lambda\right)\right| \exp \left\{-\Phi_{1}\left(u_{1} ; \lambda^{(1)}, \lambda\right)\right\} \mathrm{d} u_{1}=2 \exp \left\{\lambda\left(1-\log \left(-\lambda / \lambda^{(1)}\right)\right)\right\}<+\infty,
$$

which concludes the proof related to the existence and uniqueness of the stationary measure for the application.

Appendix D. Additional correlation graphs for exponential-type correlation functions. Here, we characterize - in a purely numerical way - the correlation function $x \mapsto \operatorname{Corr}(x)$ defined by Eq. (3.18) when other types of input correlation functions (that are, those of the Gaussian stochastic germs) are introduced. Specifically, we consider the one-dimensional application discussed in $\S 3.2 .2$, in which the correlation functions associated the two family of germs have a similar algebraic form for 
simplicity. For notational convenience, we further drop the supercripts $\ell$ and $\ell^{\prime}$ and denote the "generic" correlation function by r. We then take either an exponential correlation function, defined as

$$
\forall y \in \mathbb{R}, \quad \mathrm{r}(y)=\exp (-|y| / \mathcal{L}),
$$

or a squared exponential function, given by:

$$
\forall y \in \mathbb{R}, \quad \mathrm{r}(y)=\exp \left(-(\pi / 4) \times(y / \mathcal{L})^{2}\right) .
$$

In each case, the strictly positive parameter $\mathcal{L}$ corresponds to the spatial correlation length as defined by the left-hand side of Eq. (2.11). The plot of $x \mapsto \operatorname{Corr}(x)$ (estimated from 1000 independent realizations) for each of this correlation type is shown in Fig. D.1, where all the stochastic germs are parametrized using $\mathcal{L}=20$ $[\mathrm{mm}]$.

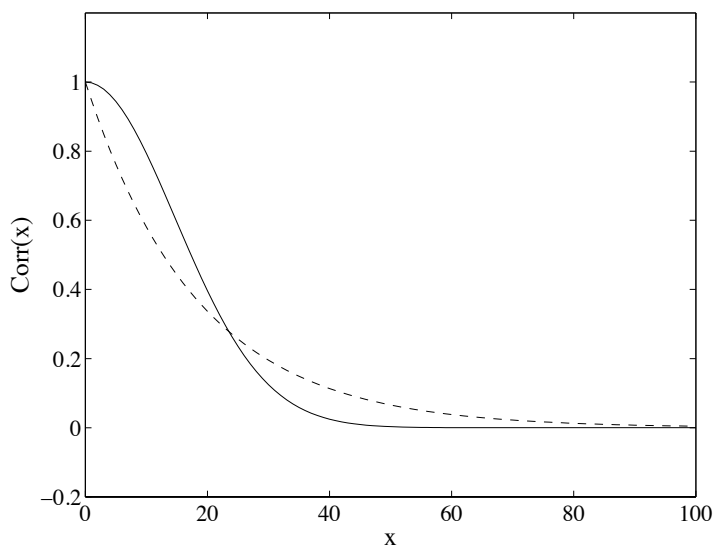

Fig. D.1. Plot of $x \mapsto \operatorname{Corr}(x)$ for different types of correlation function $\mathrm{r}$ (associated with the Gaussian random fields) and $\mathcal{L}=20$ [mm]. Dashed line: exponential type; solid line: squaredexponential type.

\section{REFERENCES}

[1] K. Acton and L. Graham-Brady. Meso-scale modeling of plasticity in composites. Computer Methods in Applied Mechanics and Engineering, 198:920-932, 2009.

[2] M. Arnst, R.G. Ghanem, and C. Soize. Identification of bayesian posteriors for coefficients of chaos expansions. Journal of Computational Physics, 229(9):3134-3154, 2010.

[3] I. Babuška, R. Tempone, and G.E. Zouraris. Solving elliptic boundary value problems with uncertain coefficients by the finite element method: the stochastic formulation. Computer Methods in Applied Mechanics and Engineering, 194:1251-1294, 2005.

[4] G. Bal and W. Jing. Corrector theory for MsFEM and HMM in random media. Multiscale Modeling \& Simulation, 9:1549-1587, 2011.

[5] R. Balian. Random matrices and information theory. Il Nuovo Cimento B, 57:183-193, 1968.

[6] A. Bóna, I. Bucataru, and M.A. Slawinski. Coordinate-free characterization of the symmetry classes of elasticity tensors. Journal of Elasticity, 87:109-132, 2007.

[7] K. Burrage, I. Lenane, and G. Lythe. Numerical methods for second-order stochastic differential equations. SIAM J. Sci. Comput., 29(1):245-264, 2007.

[8] A. Clément, C. Soize, and J. Yvonnet. Computational nonlinear stochastic homogenization using a non-concurrent multiscale approach for hyperelastic heterogeneous microstructures analysis. International Journal of Numerical Methods in Engineering, 91:799-824, 2012. 
[9] S. Das and R. Ghanem. A bounded random matrix approach for stochastic upscaling. Multiscale Modeling \& Simulation, 8(1):296-325, 2009.

[10] R. De Vogelaere. Methods of integration which preserve the contact transformation property of the hamiltonian equations. Technical Report 4, University of Notre Dame, 1956.

[11] R.V. Field and M. Grigoriu. A method for the efficient construction and sampling of vectorvalued translation random fields. Probabilistic Engineering Mechanics, 29:79-91, 2012.

[12] R.G. Ghanem and P. Spanos. Stochastic finite elements: a Spectral Approach. Springer, NewYork, 1991.

[13] S. Greene, Y. Liu, W. Chen, and W. K. Liu. Computational uncertainty analysis in multiresolution materials via stochastic constitutive theory. Computer Methods in Applied Mechanics and Engineering, 200:309-325, 2011.

[14] M. Grigoriu. Stochastic calculus: applications in science and engineering. Birkhäuser, Boston (MA), 2002.

[15] M. Grigoriu. Existence and construction of translation models for stationary non-gaussian processes. Probabilistic Engineering Mechanics, 24:545-551, 2009.

[16] J. Guilleminot, A. Noshadravan, C. Soize, and R. G. Ghanem. A probabilistic model for bounded elasticity tensor random fields with application to polycrystalline microstructures. Computer Methods in Applied Mechanics and Engineering, 200:1637-1648, 2011.

[17] J. Guilleminot and C. Soize. A stochastic model for elasticity tensors with uncertain material symmetries. International Journal of Solids and Structures, 47:3121-3130, 2010.

[18] J. Guilleminot and C. Soize. Non-Gaussian positive-definite matrix-valued random fields with constrained eigenvalues: Application to random elasticity tensors with uncertain material symmetries. International Journal of Numerical Methods in Engineering, 88:1128-1151, 2011. DOI:10.1002/nme.3212.

[19] J. Guilleminot and C. Soize. Generalized Stochastic Approach for Constitutive Equation in Linear Elasticity: A Random Matrix Model. International Journal of Numerical Methods in Engineering, 90:613-635, 2012.

[20] J. Guilleminot and C. Soize. Probabilistic modeling of apparent tensors in elastostatics: A maxent approach under material symmetry and stochastic boundedness constraints. Probabilistic Engineering Mechanics, 28:118-124, 2012. DOI:10.1016/j.probengmech.2011.07.004.

[21] J. Guilleminot and C. Soize. Stochastic modeling of anisotropy in multiscale analysis of heterogeneous materials: a comprehensive overview on random matrix approaches. Mechanics of Materials, 44:35-46, 2012. DOI:10.1016/j.mechmat.2011.06.003.

[22] E. Hairer, C. Lubich, and Wanner G. Geometric Numerical Integration. Structure-Preserving Algorithms for Ordinary Differential Equations. Springer, Berlin, 2002.

[23] B. Hiriyur, H. Waisman, and G. Deodatis. Uncertainty quantification in homogenization of heterogeneous microstructures modeled by extended finite element methods. International Journal of Numerical Methods in Engineering, 88:257-278, 2011. DOI:10.1002/nme.3174.

[24] C. Huet. Application of variational concepts to size effects in elastic heterogeneous bodies. Journal of the Mechanics and Physics of Solids, 38(6):813-841, 1990.

[25] E. T. Jaynes. Information theory and statistical mechanics. Physical Review, 106/108(4/2):620630/171-190, 1957.

[26] E. T. Jaynes. Information theory and statistical mechanics. In K. W. Ford, editor, Statistical Physics. Brandeis Lectures in Theoretical Physics 3, pages 181-218. 1963.

[27] P. E. Kloeden and E. Platen. Numerical Solution of Stochastic Differentials Equations. Springer, Berlin, 1992.

[28] P. Kree and C. Soize. Mathematics of Random Phenomena. Reidel Publishing Company, Dordrecht, Holland, 1986.

[29] I. A. Kunin. An algebra of tensor operators and its applications to elasticity. Int. J. Engng Sci., 19:1551-1561, 1981.

[30] M.M. Mehrabadi and S.C. Cowin. Eigentensors of linear anisotropic elastic materials. 43(1):1541, 1990.

[31] M.M. Mehrabadi, S.C. Cowin, and J. Jaric. Six-dimensional orthogonal tensor representation of the rotation about an axis in three dimensions. International Journal of Solids and Structures, 32(3-4):439-449, 1995.

[32] M.L. Mehta. Random Matrices (third ed.). Academic Press, New-York, 2004.

[33] A. Nouy and A. Clément. eXtended Stochastic Finite Element Method for the numerical simulation of heterogeneous materials with random material interfaces. International Journal of Numerical Methods in Engineering, 83:1312-1344, 2010. DOI:10.1002/nme.2865.

[34] M. Ostoja-Starzewski. Micromechanics as a basis of random elastic continuum approximations. Probabilistic Engineering Mechanics, 8:107-114, 1993.

[35] M. Ostoja-Starzewski. Random field models of heterogenous materials. International Journal 
of Solids and Structures, 35:2429-2455, 1998.

[36] M. Ostoja-Starzewski. Microstructural Randomness and Scaling in Mechanics of Materials. Chapman and Hall-CRC, 2008.

[37] H.M. Panayirci and G.I. Schuëller. On the Capabilities of the Polynomial Chaos Expansion Method within SFE Analysis - An Overview. Arch Comput Methods Eng, 18:43-55, 2011. DOI:10.1007/s11831-011-9058-5.

[38] F. Poirion and C. Soize. Probabilistic Methods in Applied Physics, volume 451 of Lecture Notes in Physics, chapter Numerical Methods and Mathematical Aspects for Simulation of Homogeneous and Non Homogeneous Gaussian Vector Fields, pages 17-53. SpringerVerlag, 1995.

[39] B. Puig, F. Poirion, and C. Soize. Non-gaussian simulation using hermite polynomial expansion: convergences and algorithms. Probabilistic Engineering Mechanics, 17:253-264, 2002.

[40] J. Rychlewski. On hookes law. Prikladnaya Matematika I Mekhanika, 48:303-314, 1984.

[41] J. Serra. Image analysis and Mathematical Morphology. Academic Press, London, 1982.

[42] C. E. Shannon. A mathematical theory of communication. Bell System Technical Journal, 27:379-423/623-659, 1948.

[43] C. Soize. Exact stationary response of multi-dimensional non-linear hamiltonian dynamical systems under parametric and external stochastic excitations. Journal of Sound and Vibration, 149(1):1-24, 1991.

[44] C. Soize. The Fokker-Planck Equation for Stochastic Dynamical Systems and its Explicit Steady State Solutions. World Scientific, Singapore, 1994.

[45] C. Soize. A nonparametric model of random uncertainties on reduced matrix model in structural dynamics. Probabilistic Engineering Mechanics, 15(3):277-294, 2000.

[46] C. Soize. Maximum entropy approach for modeling random uncertainties in transient elastodynamics. Journal of the Acoutical Society of America, 109(5):1979-1996, 2001.

[47] C. Soize. Non-gaussian positive-definite matrix-valued random fields for elliptic stochastic partial differential operators. Computer Methods in Applied Mechanics and Engineering, 195:26-64, 2006

[48] C. Soize. Construction of probability distributions in high dimension using the maximum entropy principle: Applications to stochastic processes, random fields and random matrices. International Journal of Numerical Methods in Engineering, 76:1583-1611, 2008.

[49] C. Soize. Tensor-valued random fields for meso-scale stochastic model of anisotropic elastic microstructure and probabilistic analysis of representative volume element size. Probabilistic Engineering Mechanics, 23:307-323, 2008.

[50] C. Soize. Identification of high-dimension polynomial chaos expansions with random coefficients for non-gaussian tensor-valued random fields using partial and limited experimental data. Computer Methods in Applied Mechanics and Engineering, 199(33-36):2150-2164, 2010.

[51] C. Soize. A computational inverse method for identification of non-gaussian random fields using the bayesian approach in very high dimension. Computer Methods in Applied Mechanics and Engineering, 200:3083-3099, 2011.

[52] C. Soize and R.G. Ghanem. Physical systems with random uncertainties: Chaos representation with arbitrary probability measure. SIAM Journal on Scientific Computing, 26(2):395410, 2004.

[53] Q.A. Ta, D. Clouteau, and R. Cottereau. Modeling of random anisotropic elastic media and impact on wave propagation. European Journal of Computational Mechanics, 19(1-23):241-253, 2010.

[54] D. Talay. Simulation and Numerical Analysis of Stochastic Differential Systems: a Review. In P. Kree and W. Wedig, editors, Probabilistic Methods in Applied Physics, volume 451 of Lecture Notes in Physics, pages 63-106. Springer-Verlag, 1995.

[55] M. Tootkaboni and L.L. Graham-Brady. A multi-scale spectral stochastic method for homogenization of multi-phase periodic composites with random material properties. International Journal of Numerical Methods in Engineering, 83:59-90, 2010. DOI:10.1002/nme.2829.

[56] S. Torquato. Random Heterogeneous Materials: Microstructure and Macroscopic Properties. Springer, New-York, 2002.

[57] E. VanMarcke. Random Fields: Analysis and Synthesis. MIT Press, Cambridge, MA, 1983.

[58] L. Verlet. Computer "Experiments" on Classical Fluids. I. Thermodynamical Properties of Lennard-Jones Molecules. Physical Review, 159(1):98-103, 1967.

[59] L. J. Walpole. Fourth-rank tensors of the thirty-two crystal classes: Multiplication tables. Proc. R. Soc. Lond. A, 391:149-179, 1984.

[60] E. Weinan, B. Engquist, X. Li, W. Ren, and E. Vanden-Eijnden. The Heterogeneous Multiscale Method: a review. Communications in Computational Physics, 2:367-450, 2007.

[61] N. Wiener. The Homogeneous Chaos. American Journal of Mathematics, 60(4):897-936, 1938. 
[62] X. F. Xu and L. Graham-Brady. A stochastic computational method for evaluation of global and local behavior of random elastic media. Computer Methods in Applied Mechanics and Engineering, 194:4362-4385, 2005. 